\title{
Herschel Spectroscopy of the Taffy Galaxies (UGC 12914/12915 = VV 254): Enhanced [C II] Emission in the Collisionally Formed Bridge
}

\author{
B. W. Peterson ${ }^{1}$, P. N. Appleton ${ }^{2}$ (D) T. Bitsakis ${ }^{3}$ (D), P. Guillard ${ }^{4}$, K. Alatalo ${ }^{5,6}$ (D) F. Boulanger ${ }^{7}$, M. Cluver ${ }^{8}$ (D) , P.-A. Duc ${ }^{9}$, \\ E. Falgarone ${ }^{7}$, S. Gallagher ${ }^{10}$, Y. Gao ${ }^{11}$, G. Helou ${ }^{2}$, T. H. Jarrett ${ }^{12}$ (D) B. Joshi ${ }^{13}$, U. Lisenfeld ${ }^{14}$, N. Lu ${ }^{15,16}$, P. Ogle ${ }^{6}$, \\ G. Pineau des Forêts ${ }^{17}$, P. van der Werf ${ }^{18}$, and C. K. Xu ${ }^{15,16}$ \\ ${ }^{1}$ University of Wisconsin-Barron County, 1800 College Drive, Rice Lake, WI 54868, USA; bradley.peterson@uwc.edu \\ ${ }^{2}$ Caltech/IPAC, MC 100-22, 1200 E. California Boulevard, Pasadena, CA 91125, USA; apple@ipac.caltech.edu \\ ${ }^{3}$ Instituto de Radioastronomía y Astrofísica, Universidad Nacional Autónoma de México, Morelia, 58089, Mexico \\ ${ }^{4}$ Sorbonne Université, CNRS, UMR 7095, Institut d'Astrophysique de Paris, 98 bis Bd Arago, F-75014 Paris, France \\ ${ }^{5}$ Carnegie Observatories, Carnegie Institute of Washington, Pasadena, CA, USA \\ ${ }^{6}$ Space Telescope Science Institute, 3700, San Martin Drive, Baltimore, MD 21218, USA \\ ${ }^{7}$ Observatoire de Paris, Université PSL, Sorbonne Université, Ecole normale supérieure, CNRS, LERMA, F-75005, Paris, France \\ ${ }^{8}$ Department of Physics and Astronomy, University of the Western Cape, Robert Sobukwe Road, Bellville, 7535, South Africa \\ ${ }^{9}$ Université de Strasbourg, CNRS, Observatoire astronomique de Strasbourg, UMR 7550, F-67000 Strasbourg, France \\ ${ }^{10}$ Department of Physics and Astronomy, University of Western Ontario, London, ON, N6A 3K7, Canada \\ ${ }^{11}$ Purple Mountain Observatory, Key Lab of Radio Astronomy, Chinese Academy of Sciences, 210008, Nanjing, People's Republic of China \\ ${ }_{12}$ Astronomy Department, University of Cape Town, Rondebosch, 7700, South Africa \\ ${ }^{13}$ School of Earth and Space Exploration, Arizona State University, Tempe, AZ 85287, USA \\ ${ }^{14}$ Departamento de Física Teorica y del Cosmos, Universidad de Granada, Spain and Instituto Carlos I de Física Teorica y Computacional, \\ Facultad de Ciencias, E-18071 Granada, Spain \\ ${ }^{15}$ National Astronomical Observatories, Chinese Academy of Sciences (CAS), Beijing 100012, People's Republic of China \\ ${ }_{16}$ China-Chile Joint Center for Astronomy, CAS, Camino El Observatorio 1515, Las Condes, Santiago, Chile \\ ${ }^{17}$ IAS (UMR 8617 du CNRS), Université de Paris Sud, F-91405 Orsay, France; LERMA (UMR 8112 du CNRS), Observatoire de Paris, \\ 61 Avenue de l'Observatoire, F-75014 Paris, France \\ ${ }^{18}$ Leiden Observatory, Leiden University, P.O. Box 9513, 2300 RA Leiden, The Netherlands \\ Received 2018 January 4; accepted 2018 January 29; published 2018 March 16
}

\begin{abstract}
Using the PACS and SPIRE spectrometers on board Herschel, we obtained observations of the Taffy galaxies (UGC 12914/12915) and bridge. The Taffy system is believed to be the result of a face-on collision between two gas-rich galaxies, in which the stellar disks passed through each other, but the gas was dispersed into a massive $\mathrm{H} \mathrm{I}$ and molecular bridge between them. Emission is detected and mapped in both galaxies and the bridge in the [C II] $157.7 \mu \mathrm{m}$ and [O I] $63.2 \mu \mathrm{m}$ fine-structure lines. Additionally, SPIRE FTS spectroscopy detects the [C I] ${ }^{3} \mathrm{P}_{2} \rightarrow{ }^{3} \mathrm{P}_{1}(809.3 \mathrm{GHz})$ and $[\mathrm{CI}]{ }^{3} \mathrm{P}_{1} \rightarrow 3 \mathrm{P}_{0}(492.2 \mathrm{GHz})$ neutral carbon lines, and weakly detects high-J CO transitions in the bridge. These results indicate that the bridge is composed of a warm multi-phase medium consistent with shock and turbulent heating. Despite low star formation rates in the bridge, the [C II] emission appears to be enhanced, reaching [C II]/FIR ratios of 3.3\% in parts of the bridge. Both the [C II] and [O I] lines show broad intrinsic multi-component profiles, similar to those seen in previous $\mathrm{CO}(1-0)$ and $\mathrm{H} \mathrm{I}$ observations. The [C II] emission shares similar line profiles with both the double-peaked H I profiles and shares a high-velocity component with single-peaked CO profiles in the bridge, suggesting that the [C II] emission originates in both the neutral and molecular phases. We show that it is feasible that a combination of turbulently heated $\mathrm{H}_{2}$ and high column-density $\mathrm{HI}$, resulting from the galaxy collision, is responsible for the enhanced [C II] emission.
\end{abstract}

Key words: galaxies: individual (UGC 12914, UGC 12915) - galaxies: interactions - intergalactic medium

\section{Introduction}

The Taffy galaxies (UGC 12914/5) are believed to be the result of a recent (25-30 Myr) face-on collision between two gas-rich disk galaxies, during which the $\mathrm{HI}$ and molecular clouds in the two disks strongly interacted, leading to the formation of a radio continuum and gas bridge between the visible galaxies (Condon et al. 1993; Struck 1997; Vollmer et al. 2012). The bridge region contains a significant fraction of the system's H I and molecular gas (Braine et al. 2003; Gao et al. 2003), and is also detected through its dust emission (Jarrett et al. 1999; Zhu et al. 2007). It is likely that the system contains gas with high levels of turbulence, and so the galaxies and bridge represent an interesting opportunity to study the heating and cooling processes in highly disturbed intergalactic gas. Similar processes may be present at high- $z$, when gas is rapidly accreted onto dark-matter halos. It is therefore important to understand nearby examples of highly turbulent systems.

Several lines of evidence show that the gas in the Taffy bridge is highly disturbed. CO (1-0) observations showed broad line widths (FWHM $\sim 200 \mathrm{~km} \mathrm{~s}^{-1}$ ) between the galaxies (Gao et al. 2003). Zhu et al. (2007) found that the strength of the $\mathrm{CO}(3-2)$ and (2-1) lines in the bridge was consistent with warm turbulent gas. Recent ALMA CO observations of bridge gas on sub-arcsec scales show a tangled web of narrow filaments with peculiar kinematics, suggestive of turbulent motions in the bridge (P. N. Appleton et al. 2018, in preparation).

Evidence for shock heating of the molecular gas also comes from the direct detection of large quantities $\left(>4.2 \times 10^{8} M_{\odot}\right)$ of warm molecular hydrogen in the bridge through Spitzer observations of the pure rotational $\mathrm{H}_{2}$ emission lines (Peterson et al. 2012). This study also showed high values of the ratios 
$L\left(\mathrm{H}_{2}\right) / L(\mathrm{PAH})$ and $L\left(\mathrm{H}_{2}\right) / L(\mathrm{FIR})$, inconsistent with photoelectric heating from photodissociation regions (PDRs) near star formation sites.

There is further evidence for shocks and turbulence in much lower-density gas. Soft X-ray emission is detected in the northern part of the bridge with Chandra (Appleton et al. 2015). The emission is consistent with million-degree hot diffuse gas heated by fast shocks. Optical emission lines from ionized gas across the bridge have also been detected in a recent optical IFU study of the Taffy (B. Joshi et al. 2018, in preparation). These observations show evidence of multipleline profiles, and LINER-like excitation consistent with gas heated by $200-300 \mathrm{~km} \mathrm{~s}^{-1}$ shocks.

In many ways, the Taffy bridge region shows striking similarities to another spatially resolved, multi-phased region: the intergalactic filament in the Stephan's Quintet compact group (hereafter SQ; Sulentic et al. 2001; Xu et al. 2003; Trinchieri et al. 2005; O'Sullivan et al. 2009). Like the Taffy, the SQ filament contains significant quantities of warm molecular gas that can be modeled by shocks and turbulent energy dissipation resulting from a galaxy collision (Appleton et al. 2006; Cluver et al. 2010). Though $\mathrm{H}_{2}$ molecules are expected to be destroyed in high-speed shocks, the models of Guillard et al. (2009) demonstrated that the molecules can reform on short timescales on dust grains that survive in highdensity clumps within a multi-phase shocked medium (mechanical heating is also suspected in translucent clouds in the Galaxy; Ingalls et al. 2011). In a recent analysis, Appleton et al. (2017) showed that the excitation of the rotational $\mathrm{H}_{2}$ lines in SQ could be modeled by a combination of low-velocity $\mathrm{C}$ - and J-shocks driven into molecular gas through supersonic turbulence originating in the large-scale collision of NGC $7318 \mathrm{~b}$ with gas in the group.

In the SQ shock, powerful emission was also detected from [C II] (Appleton et al. 2013). The authors concluded that the emission was excited by collisions with the same warm $\mathrm{H}_{2}$ that gave rise to the rotational $\mathrm{H}_{2}$ lines (see also Lesaffre et al. 2013). This energy ultimately comes from the kinetic energy of the galaxy colliding with the intergroup medium, and cascades down to small spatial scales. As in SQ, the warm $\mathrm{H}_{2}$ in the Taffy bridge appears to result from shock heating of the molecular gas. Unlike SQ, where the intruder galaxy continues to provide a source of kinetic energy to drive the turbulent dissipation, the Taffy bridge may be in a decaying turbulence phase where energy from the recent collision has been largely expended. This may explain the large amount of neutral and molecular gas in the bridge. This study was motivated by the possibility that far-IR fine-structure lines, such as [C II] and [O I], may carry additional information about the cooling of gas in the Taffy bridge.

The [C II] $157.7 \mu \mathrm{m}$ transition can be excited by a variety of mechanisms, most commonly in PDRs surrounding young stars. In these regions, the [C II] emission results from collisional excitation with warm $\mathrm{H}_{2}$ heated by photoelectrons ejected from small grains and PAHs by UV radiation (Watson 1972; Glassgold \& Langer 1974; Draine 1978; Tielens \& Hollenbach 1985; Hollenbach 1989; Bakes \& Tielens 1994). [C II] emission can extend into CO-faint molecular gas (socalled "dark molecules"; Wolfire et al. 2010), especially in starforming regions in low metallicity galaxies (e.g., Cormier et al. 2010).
However, as mentioned earlier, Peterson et al. (2012) argued that the warm $\mathrm{H}_{2}$ emission was inconsistent with photoelectric heating. Other potential collisional partners with carbon include neutral hydrogen atoms or free electrons associated with ionized gas in H II regions (Goldsmith et al. 2012). If there is sufficient energy density, cosmic rays and X-rays can also play a role. Thus, in the absence of strong star formation, the interpretation of [C II] emission is not straightforward, especially in a region like the Taffy bridge where many of these ingredients are present.

While there is little evidence of widespread star formation in the Taffy bridge, there is an extragalactic $\mathrm{H}$ II region (hereafter $\mathrm{X}-\mathrm{H}$ II) to the southwest of UGC 12915 (Figure 1). The region is faint in broadband images, but more prominent in $\mathrm{H} \alpha$ (Bushouse 1987) and $\mathrm{Pa} \alpha$ (Komugi et al. 2012). It lies in the region with the brightest $\mathrm{CO}$ emission, and is also near the position of the most luminous ultra-luminous X-ray source in the Taffy system. This supports the idea that the star formation is occurring in a young stellar association embedded in the bridge (see the discussion in Appleton et al. 2015).

Given the previous evidence of a significant luminosity in the mid-IR $\mathrm{H}_{2}$ lines, we explore the properties of the far-IR/ submillimeter emission lines from the galaxies and bridge. We obtained Herschel PACS observations in both the [C II]158 $\mu \mathrm{m}$ and [O I]63 $\mu \mathrm{m}$ emission lines, and a SPIRE FTS observation of a single pointing centered on the bridge. In Section 2, we describe the details of the Herschel PACS and SPIRE observations, and the broadband archival photometric observations. In Section 3, we present the results, including contour maps and line profiles of the [C II] and [O I] lines, as well as details of the SPIRE line detections and upper limits. We also compare the results with previously published $\mathrm{CO}(1-0)$ and $\mathrm{H}$ I data. We analyze these results in Section 4, including the modeling of spectral energy distributions (SEDs) derived from archival data, including Herschel photometry. We summarize our findings in Section 5. We assume a distance to the galaxies of $62 \mathrm{Mpc}$ based on a mean heliocentric velocity for the system of $4350 \mathrm{~km} \mathrm{~s}^{-1}$, and a Hubble constant of $70 \mathrm{~km} \mathrm{~s}^{-1} \mathrm{Mpc}^{-1}$.

\section{Observations}

\subsection{Herschel PACS Spectroscopy}

Spectroscopic observations of the Taffy were made using the Photodetector Array Camera and Spectrometer (PACS; Poglitsch et al. 2010) integral field unit (IFU) on board the Herschel Space Observatory (Pilbratt et al. 2010). ${ }^{19}$ The spectra were obtained on 2012 January 30 as part of an open time program (program ID: OT1_pappleto_1; PI: Appleton; obsids $=1342238415 / 6$ ). The PACS IFU uses a $5 \times 5$ grid of 9 !" $4 \times 9$ ". 4 spatial pixels to obtain a $47^{\prime \prime} \times 47^{\prime \prime}$ field of view. Observations of the [C II] $157.74 \mu \mathrm{m}$ and [O I]63.18 $\mu \mathrm{m}$ lines were made using a $3 \times 3$ raster map (stepsize $=38$ arcsec and repeated 4 and 5 times respectively) in chop-nod mode sampling a region of $1.83 \times 1.83 \mathrm{arcmin}^{2}$, which included both galaxies and the bridge. The angular resolution in the [C II] and [O I] lines was (FWHM) 9!"4 and 3".8, respectively, sampled onto 9 !" $4 \times 9$ !" 4 pixels. Total integration times per pointing were 23.5 and 50.0 minutes, with a total execution

\footnotetext{
19 Herschel is an ESA space observatory with science instruments provided by European-led Principal Investigator consortia and with important participation from NASA.
} 
(a)

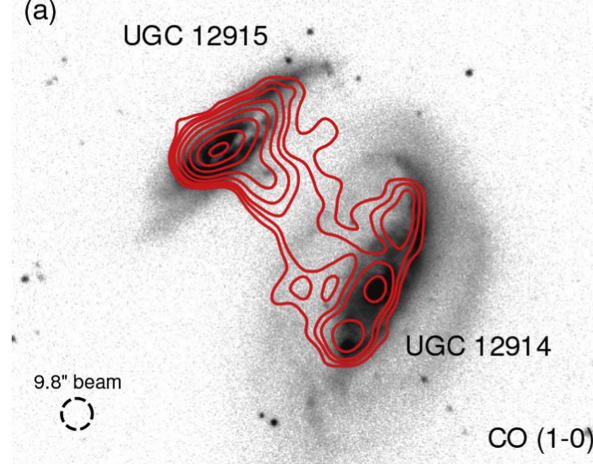

(d)

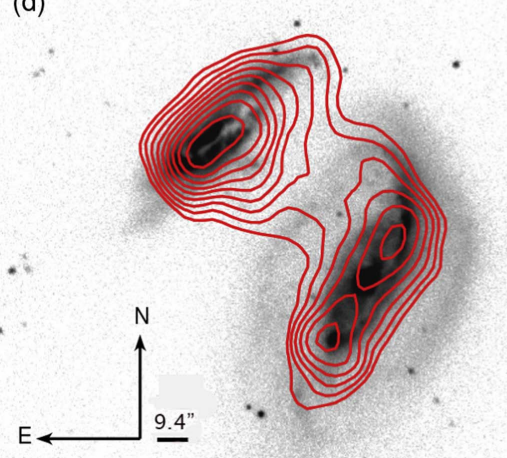

(b)

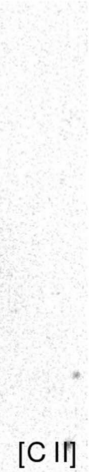

(e)

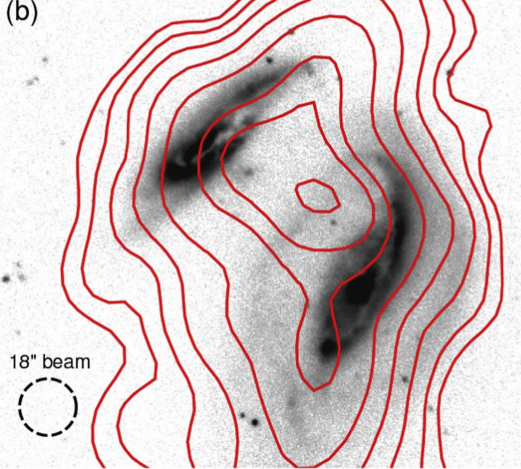

(c)
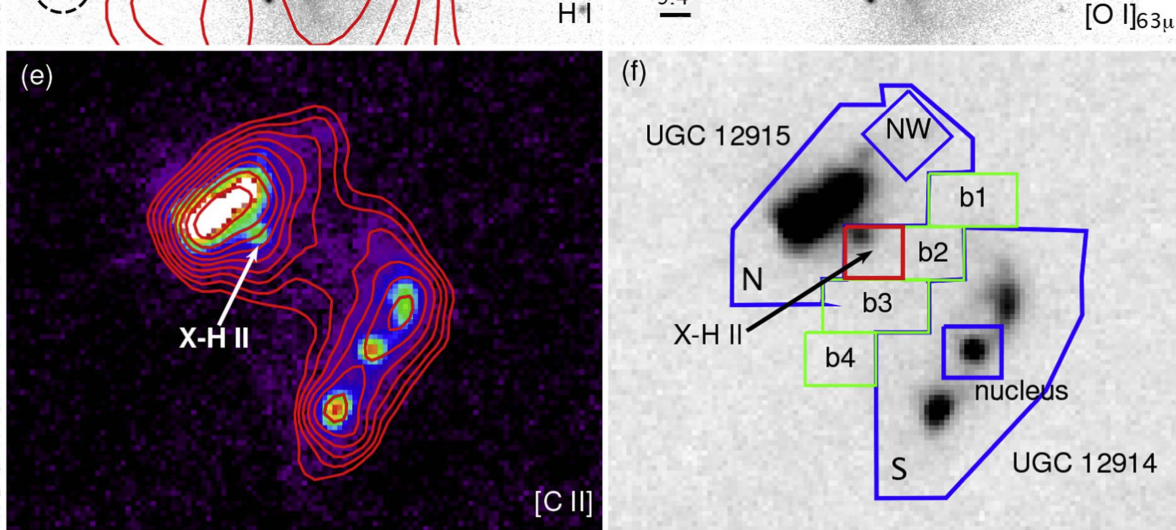

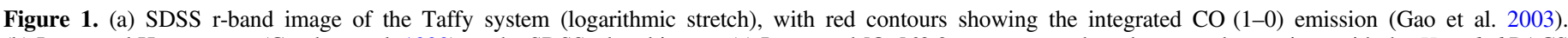

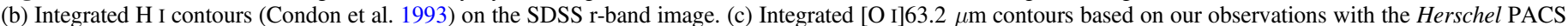

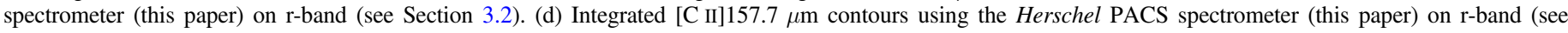

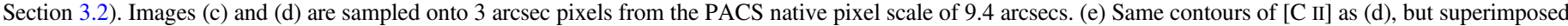

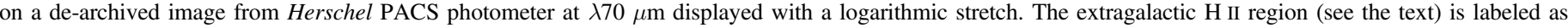

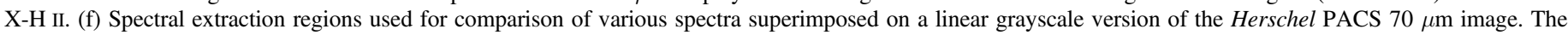

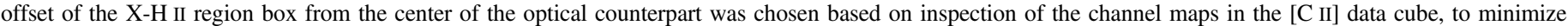
contamination of emission from the disk of UGC 12915. Some contamination will be expected.

time for the full map of 3.9 and $8.2 \mathrm{hr}$ for the [C II] and [O I] observations, respectively. ${ }^{20}$

The data were reduced using the Herschel Interactive Processing Environment (HIPE) version 15.0, and individual spectral-cube pointings were combined and regridded onto a sky projection with a pixel scale of $3^{\prime \prime}$. The spectral resolution in the $[\mathrm{CII}]$ and $[\mathrm{OI}]$ lines is $235 \mathrm{~km} \mathrm{~s}^{-1}$ and $85 \mathrm{~km} \mathrm{~s}^{-1}$ respectively. The spectra, observed in "range mode" to ensure coverage of the broad velocity field, span wavelengths from $159.4-160.8 \mu \mathrm{m} \quad\left(2570 \mathrm{~km} \mathrm{~s}^{-1}\right)$ and $64.0-64.4 \mu \mathrm{m} \quad(1965$ $\mathrm{km} \mathrm{s}^{-1}$ ), respectively, for the the [C II] and [O I] lines, centered on a redshift of $z=0.0145$.

Spectra were extracted from the final data cubes from regions covering both galaxies and the bridge, as shown in Figure 1. The four bridge regions were chosen to provide nearly complete coverage of the bridge, and to provide information about how the gas properties vary across this part of the system. Their positions are listed in Table 1. In addition, extraction regions were selected to sample X-H II, the extended emission from the northwest part of the northern galaxy (UGC 12915), and the nucleus of the southern galaxy (UGC 12914). The nuclear region of the northern galaxy would be contaminated by extended emission from the disk, so no attempt was made to extract a nuclear spectrum from this galaxy.

\footnotetext{
${ }^{20}$ Observations centered on the bridge were also attempted of the $\mathrm{OH}^{2} \Pi_{3 / 2} 5$ / 2-3/2 $\lambda$-doublet line transitions $(119.9 / 120.17 \mu \mathrm{m}$, obsid $=1342213131)$, but no emission was detected.
}

Table 1

PACS Spectral Extraction Regions

\begin{tabular}{|c|c|c|c|c|}
\hline Region & $\begin{array}{c}\text { R.A. }^{\mathrm{a}} \\
(\mathrm{J} 2000.0)\end{array}$ & $\begin{array}{c}\text { Decl. }^{\mathrm{a}} \\
(\mathrm{J} 2000.0)\end{array}$ & Size & \\
\hline UGC 12914 nuc & 00138 !" 42 & +232900" 53 & $18 ! " 3$ & $16 " .3$ \\
\hline UGC $12915 \mathrm{NW}^{\mathrm{b}}$ & 00139 " 43 & +233009".28 & $19 ! .1$ & $20 ! ! 0$ \\
\hline b1 & 00138 " 42 & +232948" 73 & $28 " .0$ & $16 " .8$ \\
\hline b2 & $\begin{array}{llll}0 & 01 & 39 ! 33\end{array}$ & +232931" 83 & $19 ! 4$ & $17 ! " 0$ \\
\hline b3 & $\begin{array}{lll}0 & 01 & 40 !\end{array}$ & +232932! 16 & $18 " .3$ & $16 " .3$ \\
\hline b4 & $00141 "$ " 47 & +232858"!34 & $22 ! 4$ & $16 " .8$ \\
\hline X-H II & $\begin{array}{lll}0 & 01 & 40 ! \prime \\
\end{array}$ & +232915!"14 & $33 " .6$ & $16 " .8$ \\
\hline
\end{tabular}

Notes.

${ }^{\mathrm{a}}$ Coordinates of center of the extraction box.

${ }^{\mathrm{b}} \mathrm{NW}$ region was also rotated by $46^{\circ} .6$.

\subsection{Herschel SPIRE Spectroscopy}

The Taffy galaxies were observed with the SPIRE Fourier Transform Spectrometer (Griffin et al. 2010) on OD 1125 (program ID: OT1_pappleto_1; PI: Appleton; obsids = 1342246980) with a total integration time of $3.7 \mathrm{hr}$ on-source. The FTS has two detector arrays, the Spectrometer Long Wave (SLW) and the Spectrometer Short Wave (SSW), which covered the long and short wavelength ranges, with a small overlap in wavelength. The complete spectrum covers the frequency range $455-1600 \mathrm{GHz}(\sim 194-671 \mu \mathrm{m})$. The FWHM of the SPIRE beam is 35 arcsec at $809.3 \mathrm{GHz}$, the rest frequency of the $[\mathrm{C} \mathrm{I}]{ }^{3} \mathrm{P}_{2} \rightarrow{ }^{3} \mathrm{P}_{1}$ line. The observations were made with a single pointing in "sparse mode" (no mapping) with a 
Table 2

SED Fit Properties

\begin{tabular}{|c|c|c|c|c|c|c|}
\hline Region & $\begin{array}{c}M_{\text {stars }} \\
10^{9} M_{\odot}\end{array}$ & $\begin{array}{c}M_{\text {dust }} \\
10^{6} M_{\odot}\end{array}$ & $\begin{array}{c}\mathrm{SFR} \\
M_{\odot} \mathrm{yr}^{-1}\end{array}$ & $\log \left(L_{\mathrm{TIR}} / L_{\odot}\right)^{\mathrm{a}}$ & $\log \left(L_{\mathrm{FIR}} / L_{\odot}\right)^{\mathrm{b}}$ & $F_{60} / F_{100}{ }^{\mathrm{c}}$ \\
\hline UGC 12915 & $43.65 \pm 4.21$ & $40.46 \pm 6.41$ & $2.77 \pm 0.30$ & $10.74 \pm 0.06$ & $10.41 \pm 0.06$ & 0.37 \\
\hline UGC 12914 & $77.62 \pm 11.51$ & $40.93 \pm 6.49$ & $1.13 \pm 0.01$ & $10.49 \pm 0.03$ & $10.09 \pm 0.03$ & 0.42 \\
\hline South Nucleus & $26.3 \pm 9.18$ & $5.15 \pm 0.23$ & $0.07 \pm 0.01$ & $9.84 \pm 0.04$ & $9.45 \pm 0.04$ & 0.33 \\
\hline UGC $12915 \mathrm{NW}$ & $2.49 \pm 0.27$ & $2.28 \pm 0.59$ & $0.03 \pm 0.01$ & $8.99 \pm 0.03$ & $8.51 \pm 0.03$ & 0.50 \\
\hline b1 & $1.42 \pm 0.02$ & $0.35 \pm 0.05$ & $0.04 \pm 0.01$ & $8.88 \pm 0.01$ & $8.53 \pm 0.01$ & 0.44 \\
\hline b2 & $2.00 \pm 0.32$ & $4.75 \pm 0.84$ & $0.04 \pm 0.01$ & $9.15 \pm 0.02$ & $8.66 \pm 0.02$ & 0.26 \\
\hline b3 & $2.41 \pm 0.26$ & $2.44 \pm 0.69$ & $0.04 \pm 0.01$ & $8.99 \pm 0.02$ & $8.52 \pm 0.02$ & 0.37 \\
\hline b4 & $1.34 \pm 0.03$ & $1.00 \pm 0.41$ & $0.01 \pm 0.01$ & $8.59 \pm 0.01$ & $8.08 \pm 0.01$ & 0.27 \\
\hline X-H II & $1.96 \pm 0.14$ & $4.98 \pm 1.11$ & $0.09 \pm 0.01$ & $9.40 \pm 0.01$ & $9.01 \pm 0.01$ & 0.32 \\
\hline
\end{tabular}

Notes.

a $3-1000 \mu \mathrm{m}$.

b $42-122 \mu \mathrm{m}$.

${ }^{\mathrm{c}} F_{\nu}(60 \mu \mathrm{m}) / F_{\nu}(100 \mu \mathrm{m})$, where $F_{\nu}$ is in Jy.

resolving power ranging between $372<R<1288$ over the full range of the spectra. The central beam was pointed at the bridge center at $\alpha(\mathrm{J} 2000)=00^{\mathrm{h}} 01^{\mathrm{m}} 40^{\mathrm{s}} 3, \quad \delta(\mathrm{J} 2000)=23^{\circ} 29^{\prime} 22^{\prime \prime} 0$. Although other beams, apart from the central one, intersect with parts of the two galaxies, the emphasis in the paper is on the bridge, and so the other spectra will not be described in the current paper.

SPIRE data reduction was performed using HIPE 15. The observed lines were extracted using a custom routine, after subtracting the appropriate background, which was observed on that date and at the same resolution. This routine implemented a Sinc function to match the line profiles.

\subsection{Multi-wavelength Broadband Photometry}

Broadband multi-wavelength coverage of the Taffy system was used to construct SEDs for several extraction regions (see the later discussion). These data were extracted from various archives, and included UV coverage from Galaxy Evolution Explorer (Martin et al. 2005), visible wavelengths from the Sloan Digital Sky Survey DR 7 (SDSS ${ }^{21}$ ), mid-IR from Spitzer IRAC and MIPS (Fazio et al. 2004; Rieke et al. 2004; Werner et al. 2004, from NASA's Infrared Science Archive-IRSA ${ }^{22}$ ), and far-IR PACS photometry from the Herschel Science Archive. ${ }^{23}$

To estimate the physical properties of the galaxies, we have used MAGPHYS (da Cunha et al. 2008, 2010) to fit the observed SEDs with sets of model templates. This code accounts for the global energy balance between the optical and the infrared and uses a Bayesian approach that draws from a large library of random models encompassing all plausible parameter combinations to derive the probability distribution function of various galaxy parameters, such as the stellar masses, star formation rates (SFRs), and both the total- and farinfrared luminosity for each of the regions. These values are listed in Table 2.

\footnotetext{
$\overline{21}$ Funding for SDSS-III has been provided by the Alfred P. Sloan Foundation, the Participating Institutions, the National Science Foundation, and the U.S. Department of Energy Office of Science. The SDSS-III website is http://www. sdss3.org/.

22 This research has made use of the NASA/IPAC Infrared Science Archive, which is operated by the Jet Propulsion Laboratory, California Institute of Technology, under contract with the National Aeronautics and Space Administration.

${ }^{23}$ http://archives.esac.esa.int/hsa/whsa/ and IRSA.
}

\section{Results}

\subsection{Spatial Distribution of the [C II] and [O I] Emission}

To provide background on our observations, we present CO (Gao et al. 2003) and H I (Condon et al. 1993) contours of the Taffy system superimposed on an optical image of the galaxies in Figures 1(a) and (b). The CO interferometer observations, which have a similar spatial resolution to the PACS [C II] observations, show a clear bridge extending from UGC 12915 to UGC 12914. The H I observations, with much poorer spatial resolution, show a large concentration of $\mathrm{HI}$ peaking between the galaxies. In addition to the difference in spatial resolution, the main centroid of the $\mathrm{HI}$ emission may be offset toward the northwest compared with the $\mathrm{CO}$ emission.

Figure 1(c) presents integrated emission contours of [O I] $63 \mu \mathrm{m}$ derived from the PACS observations. The inner disks of both UGC 12915 and UGC 12914 are strongly detected in [O I]. A ridge of emission in UGC 12915 follows the dark dust lanes in the disk, and extends toward the northwest, where the emission becomes more extended. In UGC 12914, the emission is concentrated toward the nucleus, and in two bright regions further out in the disk. Clumpy emission from [OI] is present between the galaxies, including the X-H II region, and a prominent clump between the galaxies. Fainter emission is present in the bridge, but this is not well represented in the integrated map.

Figures 1(d) and (e) show the distribution of [C II] emission superimposed on both the optical image and the $70 \mu \mathrm{m}$ PACS dust continuum image obtained from the Herschel Science Archive. The [C II] distribution is similar to that of the [O I], though there are some differences. For example, the [C II] emission appears to be strong relative to the [O I] in the northwest part of UGC 12915. Furthermore, the nucleus of UGC 12914 seems more prominent in the [O I] emission, as compared with [C II]. The [C II] is also well-correlated with the faint dust emission, including faint emission in the bridge. It is notable that the southern boundary of UGC 12914 is very sharply defined, similar to the $\mathrm{CO}$ and radio continuum distributions (the latter is not shown here, but see Condon et al. 1993). The emission does not extend into the outer southern ring of UGC 12914, indicating that the ISM of this 


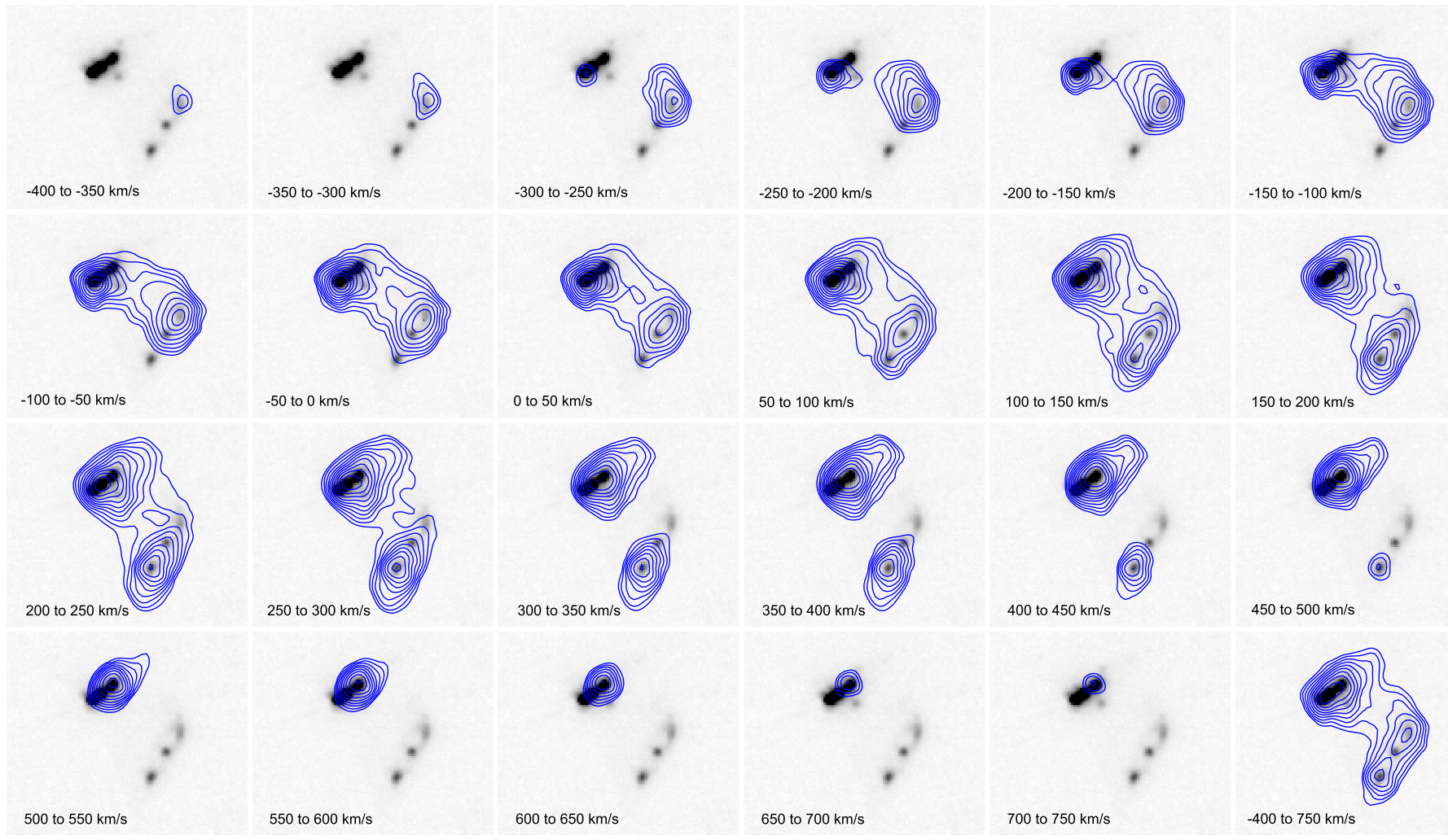

Figure 2. [C II] velocity contours over the PACS $70 \mu \mathrm{m}$ image. The contour levels begin at $3.0 \mathrm{Jy} \mathrm{km} \mathrm{s} \mathrm{s}^{-1}$ and increase successively by a factor of $\sqrt{2}$. The last panel is integrated over the entire velocity range with contour levels beginning at $20 \mathrm{Jy} \mathrm{km} \mathrm{s} \mathrm{s}^{-1}$ and increasing successively by a factor of $\sqrt{2}$. Zero velocity is equal to $4350 \mathrm{~km} \mathrm{~s}^{-1}$ heliocentric.

galaxy has been severely stripped from the disk by the collision with UGC 12915.

Based on the gas distribution, several spectral extraction boxes were defined, as shown in Figure 1(f). The large polygons are intended to capture all of the emission from the galaxies. The bridge is broken up into four regions b1-b4 to allow us to look for variations in the gas properties of the bridge. We also extracted a spectrum from the unusual extragalactic X-H II region, and the nucleus of UGC 12914. The UGC 12915 nucleus could not be isolated in either the [C II] or $70 \mu \mathrm{m}$ emission and was not extracted. Instead, we extracted the extended emission in the northwest part of UGC 12915. This is a region where the underlying far-IR continuum is weak, but the [C II] emission is quite pronounced.

\subsection{Velocity Distribution of [C II] and [O I] Emission}

Figure 2 shows velocity contour maps for the [C II] line. As previously noted by many authors, the two galaxies appear to be counter-rotating. The emission in UGC 12914 is from $v \sim-400$ to $500 \mathrm{~km} \mathrm{~s}^{-1}$, with negative velocities at the northern end of the galaxy and positive toward the south. ${ }^{24}$ The emission at the southern end of the galaxy extends along the visible spiral arm farther than is apparent from the integrated contours of Figure 1, with extended emission from $v \sim 200$ to $400 \mathrm{~km} \mathrm{~s}^{-1}$. On the other hand, UGC 12915 is detected from $v \sim-300$ to $750 \mathrm{~km} \mathrm{~s}^{-1}$ with negative velocities toward the south and positive toward the north: the opposite of UGC 12914. The emission peak is confined to

\footnotetext{
${ }^{24}$ All Velocities are heliocentric and relative to a radial velocity of $4350 \mathrm{~km} \mathrm{~s}^{-1}$.
}

the same compact area as the $70 \mu \mathrm{m}$ emission at all velocities. The extended emission in the northwest part of the galaxy is evident from $v \sim 150$ to $450 \mathrm{~km} \mathrm{~s}^{-1}$. This is a wider velocity range than the emission along the southern arm of UGC 12914.

Emission in the bridge is detected from -300 to $350 \mathrm{~km} \mathrm{~s}^{-1}$, starting just south of the northern arm of UGC 12914 and moving across the bridge toward the center of UGC 12915 at increasing velocities. The X-H II region near UGC 12915 appears at velocities from $v \sim-100$ to $300 \mathrm{~km} \mathrm{~s}^{-1}$. While much of the emission from X-H II appears at the same velocities as the diffuse bridge emission, it is also strong from 200 to $300 \mathrm{~km} \mathrm{~s}^{-1}$, where the diffuse bridge emission has largely faded.

[O I] velocity contours are presented in Figure 3. UGC 12915 is detected from $v=-250$ to $600 \mathrm{~km} \mathrm{~s}^{-1}$ and, as in [C II], shows extended emission to the northwest. Clumpy extended emission appears to extend along the northwest disk of UGC 12915, and extend into the bridge from $\sim 0$ to $200 \mathrm{~km} \mathrm{~s}^{-1}$. Starting at $\sim 200 \mathrm{~km} \mathrm{~s}^{-1}$, and continuing through $\sim 350 \mathrm{~km} \mathrm{~s}^{-1}$, the emission extends well past the northern arm. The X-H II region is better defined in the [O I] maps than in [C II]. The narrow northwestern arm of UGC 12914 extends out of the disk and into the bridge at velocities of -300 to $-200 \mathrm{~km} \mathrm{~s}^{-1}$.

\subsection{A Comparison between the [C II] and [O I] Spectra, and That of $\mathrm{CO}(1-0)$ and $\mathrm{HI}$}

Figure 4 shows the profiles of the [C II] (black lines) and [O I] (green lines) lines for each of the extraction regions. The [C II] is strong (peak flux densities of 50 and $25 \mathrm{Jy}$ integrated 


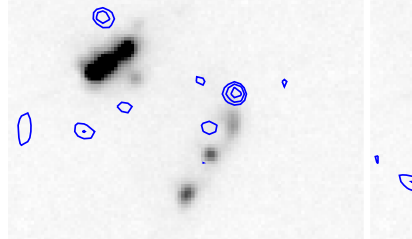

-35 to $-300 \mathrm{~km} / \mathrm{s}$
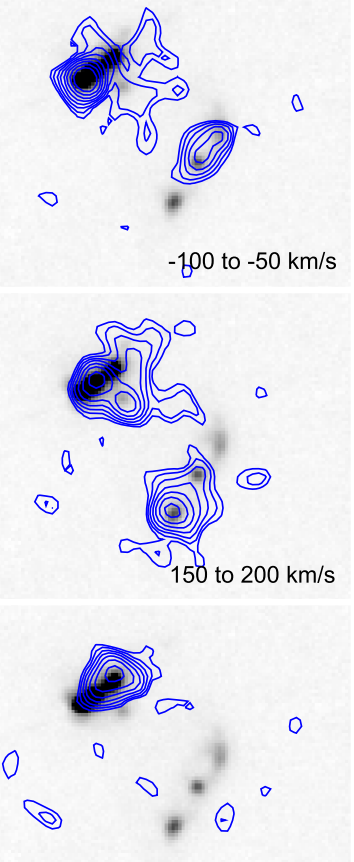

400 to $450 \mathrm{~km} / \mathrm{s}$

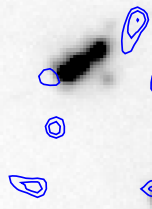

-396 to $-250 \mathrm{~km} / \mathrm{s}$

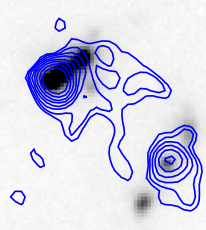

-50 to $0 \mathrm{~km} / \mathrm{s}$
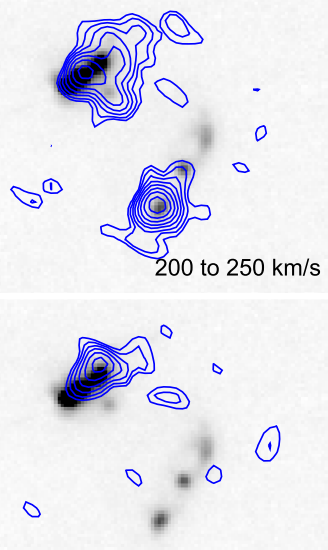

450 to $500 \mathrm{~km} / \mathrm{s}$
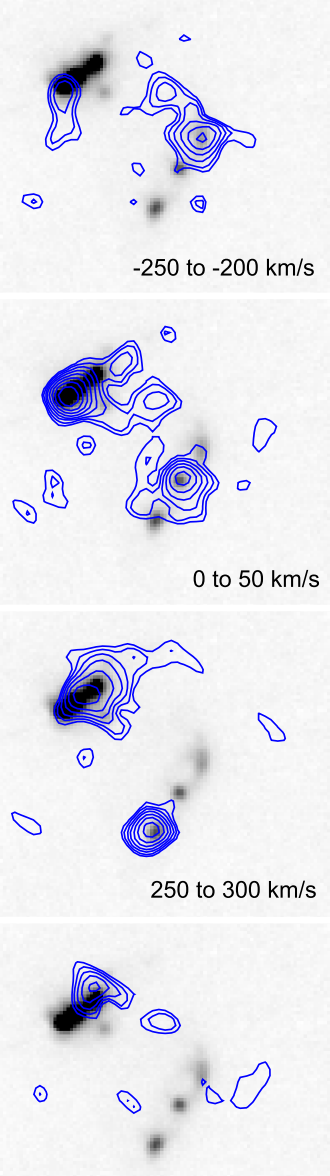

500 to $550 \mathrm{~km} / \mathrm{s}$
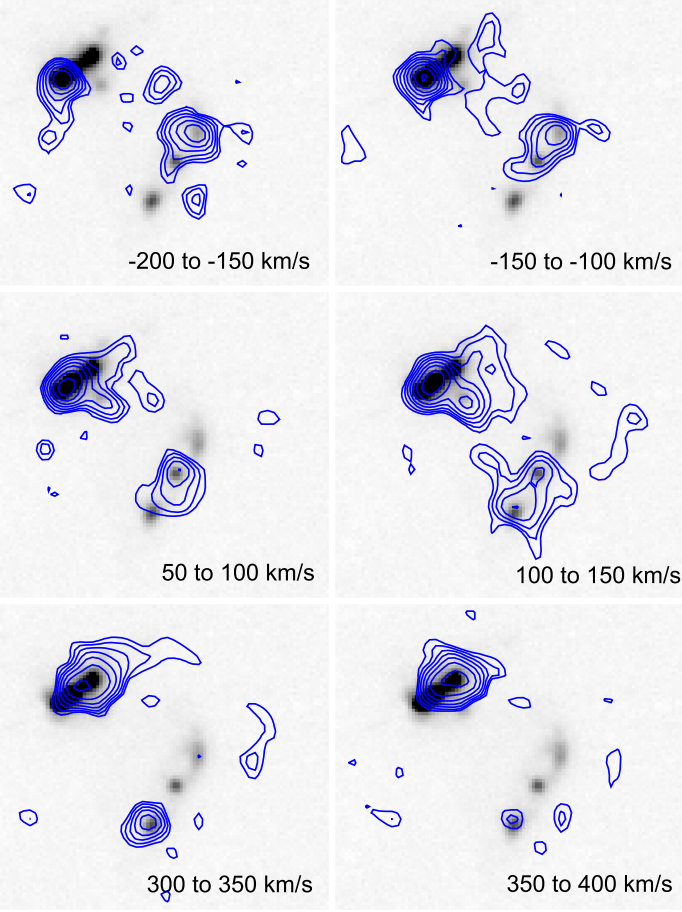

100 to $150 \mathrm{~km} / \mathrm{s}$
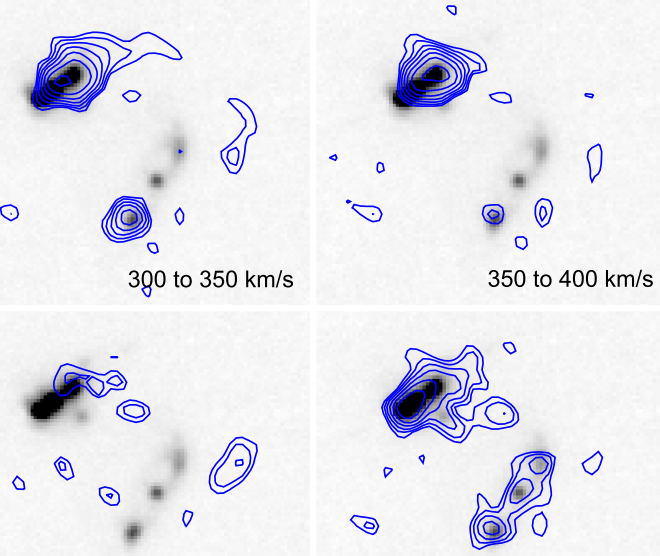

550 to $600 \mathrm{~km} / \mathrm{s}$

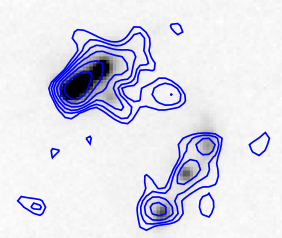

-350 to $600 \mathrm{~km} / \mathrm{s}$

Figure 3. [O I] velocity maps over PACS $70 \mu \mathrm{m}$ image. The contour levels begin at $1.0 \mathrm{Jy} \mathrm{km} \mathrm{s}{ }^{-1}$ and increase successively by a factor of $\sqrt{2}$. The last panel is integrated over the entire velocity range with contour levels beginning at $10 \mathrm{Jy} \mathrm{km} \mathrm{s}{ }^{-1}$ and increasing successively by a factor of $\sqrt{2}$. Zero velocity is equal to $4350 \mathrm{~km} \mathrm{~s}^{-1}$ heliocentric.

[C II] and [O I] Line Profiles
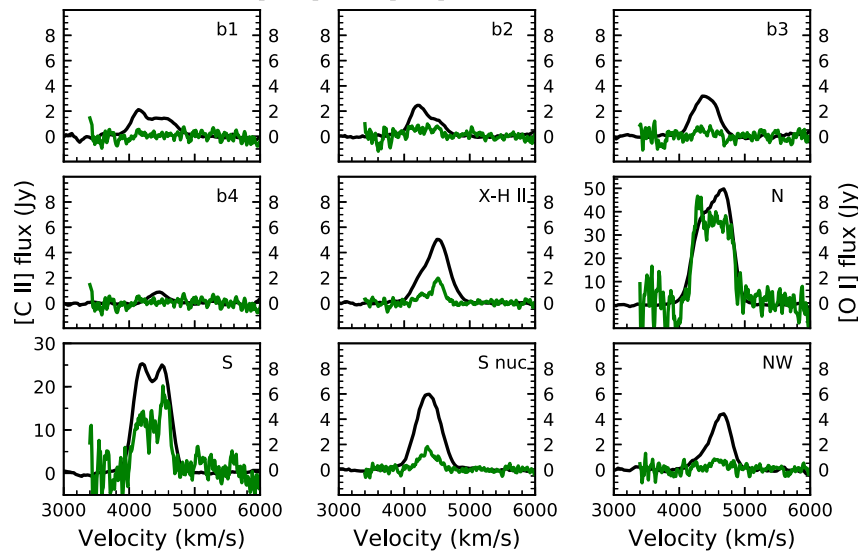

Figure 4. [C II] $157.7 \mu \mathrm{m}$ line profiles (black) and [O I]63.1 $\mu \mathrm{m}$ (green) with spectral resolutions of $235 \mathrm{~km} \mathrm{~s}^{-1}$ and $85 \mathrm{~km} \mathrm{~s}^{-1}$ respectively. The extraction regions are shown in Figure 1 and their positions and sizes are listed in Table 1.

over the two galaxies; labeled N and S for UGC 12915 and UGC 12914 respectively), and weaker in the bridge and the $\mathrm{X}-\mathrm{H}$ II region (in the peak flux range of a few jansky; labeled b1 - b4 and X-H II). The bridge and X-H II extractions show very broad line profiles that suggest multiple components. Similar, though weaker and noisier, broad profiles are also seen in the $[\mathrm{O} \mathrm{I}]$ extractions (green lines) shown in Figure 4. The [O I]63 $\mu \mathrm{m}$ observations have higher resolving power $\left(85 \mathrm{~km} \mathrm{~s}^{-1}\right)$ and are better able to resolve the complex velocity structure of the bridge. For example, the X-H II region spectrum in [O I] shows a narrower high-velocity feature and a broader low-velocity feature, consistent with the lower resolution $[\mathrm{C} \mathrm{II}]$ profile. The $[\mathrm{O} \mathrm{I}]$ emission is weakly detected at positions b2 and b3, is marginally detected in b1, and is not detected in b4. As in the case of the [C II] profile, the [O I] emission at bridge positions b1 and b2 shows multiple components, whereas b3 is better represented by a single component.

The line fluxes and velocity components for the [C II] and [O I] lines are given in Tables 3 and 4, respectively, with line ratios provided in Table 5. Line fluxes were measured using the ISO Spectral Analysis Package (Sturm et al. 1998). Each line was measured using either a single or double line fit to these spectra. The two components usually provided a better match to $[\mathrm{C} \mathrm{II}]$ profiles. There were, however, cases where the data were fit with a single component, such as b2 and b3, where the [O I] is weak. In b1 and b4, the [O I] emission is sufficiently weak that we are only able to provide $3 \sigma$ upper limits.

We note that in the bridge position $\mathrm{b} 3$, the observed line ratio of $[\mathrm{C} \mathrm{II}] /[\mathrm{OI}]=2.75 \pm 0.66$, which is similar to that seen in several of in the intergalactic shocked regions in the SQ system 
Table 3

PACS [C II] Measurements

\begin{tabular}{|c|c|c|c|c|c|c|c|c|c|}
\hline Region & $\begin{array}{l}\text { Line Flux } \\
\left(10^{-17} \mathrm{~W} \mathrm{~m}^{-2}\right)\end{array}$ & $\begin{array}{c}V_{1} \\
\text { Heliocentric } \\
\left(\mathrm{km} \mathrm{s}^{-1}\right)\end{array}$ & $\begin{array}{c}\Delta V_{1}^{\mathrm{a}} \\
\mathrm{FWHM} \\
\left(\mathrm{km} \mathrm{s}^{-1}\right)\end{array}$ & $\begin{array}{c}\Delta V 1^{\mathrm{b}} \\
\mathrm{FWHM} \\
\left(\mathrm{km} \mathrm{s}^{-1}\right)\end{array}$ & $\begin{array}{l}V_{1} \text { Frac }^{\mathrm{c}} \\
\text { (percent) }\end{array}$ & $\begin{array}{c}V_{2} \\
\text { Heliocentric } \\
\left(\mathrm{km} \mathrm{s}^{-1}\right)\end{array}$ & $\begin{array}{c}\Delta V_{2}^{\mathrm{a}} \\
\text { FWHM } \\
\left(\mathrm{km} \mathrm{s}^{-1}\right)\end{array}$ & $\begin{array}{c}\Delta V_{1}^{\mathrm{b}} \\
\text { FWHM } \\
\left(\mathrm{km} \mathrm{s}^{-1}\right)\end{array}$ & $\begin{array}{l}V_{2} \text { Frac }^{\mathrm{c}} \\
\text { (percent) }\end{array}$ \\
\hline UGC 12915 & $186.3 \pm 19.1$ & 4369 & 369 & 284 & 50 & 4692 & 306 & 195 & 50 \\
\hline UGC 12914 & $96.4 \pm 10.2$ & 4188 & 297 & 181 & 52 & 4516 & 287 & 165 & 48 \\
\hline UGC 12914 nuc & $17.3 \pm 1.8$ & 4367 & 425 & 354 & 100 & $\ldots$ & $\ldots$ & $\ldots$ & $\ldots$ \\
\hline UGC $12915 \mathrm{NW}$ & $11.6 \pm 1.2$ & 4497 & 484 & 423 & 49 & 4676 & 278 & 148 & 51 \\
\hline b1 & $7.7 \pm 1.0$ & 4141 & 249 & 82 & 41 & 4509 & 445 & 377 & 59 \\
\hline b2 & $6.9 \pm 0.9$ & 4214 & 278 & 149 & 68 & 4508 & 276 & 145 & 32 \\
\hline b3 & $9.1 \pm 1.0$ & 4323 & 334 & 237 & 65 & 4533 & 271 & 135 & 35 \\
\hline b4 & $1.8 \pm 0.2$ & 4343 & 318 & 215 & 100 & $\ldots$ & $\ldots$ & $\ldots$ & $\ldots$ \\
\hline H II & $14.8 \pm 1.5$ & 4382 & 457 & 392 & 100 & $\ldots$ & $\ldots$ & $\ldots$ & $\ldots$ \\
\hline
\end{tabular}

Notes.

${ }^{\mathrm{a}}$ FWHM from the observed line profile.

b Intrinsic FWHM after deconvolution with the instrument profile.

${ }^{\mathrm{c}}$ Fraction of total flux in each component.

(Appleton et al. 2013), and with similar [C II] surface intensity. By analogy with that work, we estimate similar derived properties to that diffuse gas $\left(T \sim 200 \mathrm{~K} ; n \sim 10^{3} \mathrm{~cm}^{-3}\right)$ in the Taffy bridge (see Figures 6 and 7 of that paper).

The emission in [C II] from the NW-disk extraction region in UGC 12915 is quite strong and broad, given its weak far-IR emission (see Figure 1(f)), and is much weaker in [O I]. It is possible that this emission is bridge material superimposed on the northern disk, as it shares similar properties to other material in the bridge. X-ray emission, which we suggested was shock-heated gas left over from the initial collision between the Taffy galaxies (Appleton et al. 2015), also occupies this region of the northwest disk of UGC 12915 and the bridge.

Both the [C II] and [O I] profiles of the nucleus of UGC 12914 (labeled S-nuc in Figure 4) show broad lines (FWHM 354 and $288 \mathrm{~km} \mathrm{~s}^{-1}$, respectively, for [C II] and [O I] after deconvolving with the instrument profile). The Chandra X-ray spectral index measurements of Appleton et al. (2015) showed possible evidence for a weak low-luminosity AGN in this galaxy.

To compare the PACS spectra of the extracted regions with the Condon et al. (1993) and CO data from BIMA CO (1-0) (Gao et al. 2003), we obtained calibrated data cubes of both sets of observations and performed extractions over similar areas to those of Figure 1. Figure 5 shows the emission profiles of [C II] with $\mathrm{HI}$ and $\mathrm{CO}(1-0)$, and Figure 6 compares the [O I] and $\mathrm{CO}(1-0)$ line profiles. The $\mathrm{CO}(1-0)$ spatial resolution $(9$ !! $8 \times 9$ !. 8$)$ is similar to that of the $[\mathrm{C} \mathrm{II}]$, but the $\mathrm{H}$ I data has much poorer resolution $\left(18^{\prime \prime} \times 18^{\prime \prime}\right)$, and so this must be taken into account. All of the Herschel spectral extraction boxes are large enough to include at least one beam of the H I VLA data. Nevertheless, these latter profiles are likely to be more affected by resolution effects than the other spectra. In some of our comparisons, we have convolved the $\mathrm{CO}$ and $[\mathrm{C} \mathrm{II}]$ data to the same resolution as the $\mathrm{HI}$ data.

There are several regions in the Taffy bridge where the [C II], $\mathrm{HI}$, and $\mathrm{CO}$ (1-0) profiles differ, even after taking into account the differing resolutions. The biggest differences are between the $\mathrm{CO}$ and the $\mathrm{HI}$ in regions b2, b3, and in X-H II. The $\mathrm{HI}$ is double-peaked, with a low- and high-velocity component that is similar to the [C II] profiles, whereas the $\mathrm{CO}$ is single-peaked with the high-velocity component being dominant. This is particularly striking in the X-H II spectrum, where the
CO (1-0) profile shows almost no emission in the low-velocity component, whereas the $\mathrm{HI}$ and [C II] profiles exhibit both components. In region $\mathrm{X}-\mathrm{H} \mathrm{II}$, the [O I] emission is more strongly peaked on the high-velocity component, corresponding with the $\mathrm{CO}$, but also shows weaker emission in the low-velocity component of the double profile. In other regions of the bridge, the situation is less clear-cut. For example, in b2 and $\mathrm{b} 3$, the $\mathrm{CO}$ contains a bright high-velocity component, but a weaker low-velocity component, both of which are represented in [C II] and weakly in [O I].

The fact that the $[\mathrm{C} \mathrm{II}]$ and $\mathrm{H}$ I share similar double-peaked line-shapes in parts of the bridge, but that the CO (1-0)emitting gas primarily dominates the higher-velocity peak, shows that the two kinematic components contain a different mix of $\mathrm{HI}$ and CO-bright $\mathrm{H}_{2}$ (we cannot, of course, rule out CO-faint $\mathrm{H}_{2}$; Wolfire et al. 2010). Both velocity components also contain an unknown (but potentially large) fraction of midIR-emitting warm molecular hydrogen (the Spitzer IRS observations did not have sufficient resolution to separate the high- and low-velocity components; Peterson et al. 2012). It is therefore very likely that both neutral and molecular hydrogen contribute to the collisional excitation of the [C II $] 157 \mu \mathrm{m}$ line in the presence of a small degree of UV ionization. We will return to this point in Section 4.

\subsection{The SPIRE Spectrum of the Bridge Center}

Figure 7 shows the spectrum of the bridge split into several pieces to zoom in on the relevant detected lines. Several lines are detected, including the neutral carbon lines $[\mathrm{C} \mathrm{I}]{ }^{3} \mathrm{P}_{2} \rightarrow{ }^{3} \mathrm{P}_{1}$ (rest frequency $809.3 \mathrm{GHz}=370.4 \mu \mathrm{m}$ ), $[\mathrm{C} \mathrm{I}]{ }^{3} \mathrm{P}_{1} \rightarrow{ }^{3} \mathrm{P}_{0}$ (rest frequency $492.2 \mathrm{GHz}=609.1 \mu \mathrm{m}$; hereafter $[\mathrm{CI}](2-1)$ and [C I] (1-0) respectively). The ${ }^{12} \mathrm{CO}(6-5)$ and ${ }^{12} \mathrm{CO}(5-4)$ are weakly detected at a lower level of significance. The ${ }^{12} \mathrm{CO}$ (4-3) line appears to be stronger; however, it is very close to the band edge so its existence must be treated with caution. We also measured a $3 \sigma$ upper limit for the [N II] $205 \mu \mathrm{m}$ line. The line measurements are presented in Table 6.

The detection of two [C I] lines is perhaps not surprising given that they likely trace diffuse molecular gas with similar density to that of the CO (1-0) emitting gas (Papadopoulos \& Greve 2004). The critical densities of the [C I] (2-1) and (1-0) are 500 and $1000 \mathrm{~cm}^{-3}$, respectively, which is very similar to that of $\mathrm{CO}(1-0)\left(440 \mathrm{~cm}^{-3}\right)$. Interestingly, the ratio of the 
Table 4

PACS [O I] Measurements

\begin{tabular}{|c|c|c|c|c|c|c|c|c|c|}
\hline Region & $\begin{array}{c}\text { Line Flux } \\
\left(10^{-17} \mathrm{~W} \mathrm{~m}^{-2}\right)\end{array}$ & $\begin{array}{c}V_{1} \\
\text { Heliocentric } \\
\left(\mathrm{km} \mathrm{s}^{-1}\right)\end{array}$ & $\begin{array}{c}\Delta V_{1}^{\mathrm{a}} \\
\mathrm{FWHM} \\
\left(\mathrm{km} \mathrm{s}^{-1}\right)\end{array}$ & $\begin{array}{c}\Delta V 1^{\mathrm{b}} \\
\mathrm{FWHM} \\
\left(\mathrm{km} \mathrm{s}^{-1}\right)\end{array}$ & $\begin{array}{l}V_{1} \operatorname{frac}^{\mathrm{c}} \\
\text { (percent) }\end{array}$ & $\begin{array}{c}V_{2} \\
\text { Heliocentric } \\
\left(\mathrm{km} \mathrm{s}^{-1}\right)\end{array}$ & $\begin{array}{c}\Delta V_{2}^{\mathrm{a}} \\
\mathrm{FWHM} \\
\left(\mathrm{km} \mathrm{s}^{-1}\right)\end{array}$ & $\begin{array}{c}\Delta V_{1}^{\mathrm{b}} \\
\mathrm{FWHM} \\
\left(\mathrm{km} \mathrm{s}^{-1}\right)\end{array}$ & $\begin{array}{l}V_{2} \mathrm{Frac}^{\mathrm{c}} \\
\text { (percent) }\end{array}$ \\
\hline UGC 12915 & $67.7 \pm 8.4$ & 4288 & 169 & 146 & 27 & 4516 & 165 & 142 & 73 \\
\hline UGC 12914 nuc & $7.8 \pm 0.8$ & 4370 & 300 & 288 & 100 & $\ldots$ & $\ldots$ & $\ldots$ & $\ldots$ \\
\hline UGC $12915 \mathrm{NW}$ & $4.3 \pm 0.5$ & 4598 & 215 & 198 & 100 & $\cdots$ & $\cdots$ & $\cdots$ & $\ldots$ \\
\hline b1 & $\leqslant 1.47$ & $\ldots$ & $\ldots$ & $\ldots$ & $\ldots$ & $\ldots$ & $\cdots$ & $\cdots$ & $\cdots$ \\
\hline b4 & $\leqslant 3.0$ & $\ldots$ & $\ldots$ & $\ldots$ & $\ldots$ & $\ldots$ & $\ldots$ & $\ldots$ & $\ldots$ \\
\hline H II & $7.8 \pm 0.8$ & 4267 & 176 & 155 & 25 & 4518 & 165 & 142 & 75 \\
\hline
\end{tabular}

Notes.

${ }^{\text {a }}$ FWHM from the observed line profile.

${ }^{\mathrm{b}}$ Intrinsic FWHM after deconvolution with the instrument profile.

${ }^{c}$ Fraction of total flux in each component.

Table 5

Line Ratios

\begin{tabular}{lccc}
\hline \hline Region & {$[\mathrm{C} \mathrm{II}] /[\mathrm{O} \mathrm{I}]$} & {$[\mathrm{C} \mathrm{II}] / \mathrm{CO}^{\mathrm{a}}$} & $\begin{array}{c}{[\mathrm{C} \text { II }] / L_{\mathrm{FIR}}{ }^{\mathrm{b}}} \\
\text { Percent }\end{array}$ \\
\hline UGC 12915 & $2.75 \pm 0.44$ & $669 \pm 67$ & $0.86 \pm 0.09$ \\
UGC 12914 & $2.58 \pm 0.39$ & $465 \pm 47$ & $0.94 \pm 0.10$ \\
UGC 12914 nuc & $2.23 \pm 0.32$ & $403 \pm 40$ & $0.74 \pm 0.07$ \\
UGC 12915 NW & $2.71 \pm 0.40$ & $1286 \pm 129$ & $4.35 \pm 0.44$ \\
b1 & $\geqslant 5.22$ & $909 \pm 91$ & $2.73 \pm 0.36$ \\
b2 & $1.39 \pm 0.24$ & $348 \pm 35$ & $1.80 \pm 0.24$ \\
b3 & $2.75 \pm 0.66$ & $218 \pm 22$ & $3.31 \pm 0.35$ \\
b4 & $\geqslant 0.59$ & $191 \pm 19$ & $1.78 \pm 0.20$ \\
X-H II & $1.90 \pm 0.27$ & $236 \pm 24$ & $1.72 \pm 0.18$ \\
\hline
\end{tabular}

Notes.

a The PACS [C II] and BIMA CO beams have comparable sizes of 9 !" $4 \times 9$ !. 4 and 9 !. $9 \times 9$ ! 7 , respectively. The uncertainties in the CO fluxes were assumed to be $10 \%$.

b $42-122 \mu \mathrm{m}$.

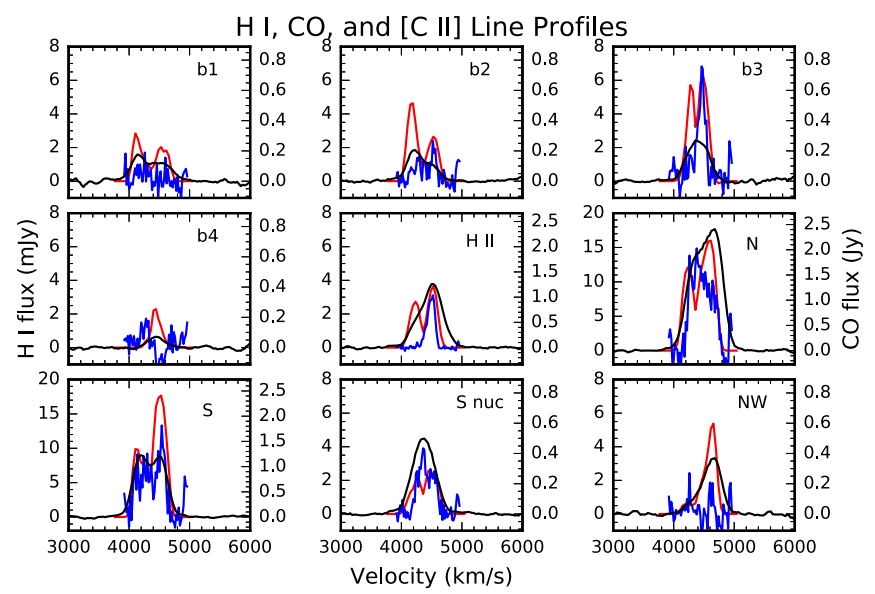

Figure 5. Line profiles for $\mathrm{H} \mathrm{I}$ (red), $\mathrm{CO}(1-0)$ (blue), and [C II] (black). The [C II] units are arbitrary; for values, see Figure 4. The extraction regions are shown in Figure 1 and their positions and sizes are listed in Table 1. The $\mathrm{H}$ I data have a spectral resolution of $42.4 \mathrm{~km} \mathrm{~s}^{-1}$, while the resolution in CO is $20 \mathrm{~km} \mathrm{~s}^{-1}$.

[C I] $(2-1)$ and (1-0) lines, $R_{[\mathrm{C} \mathrm{I}]}=0.7 \pm 0.1$, is an outlier when compared with samples of normal and luminous infrared galaxies (LIRGs), where typical galaxies show values in the

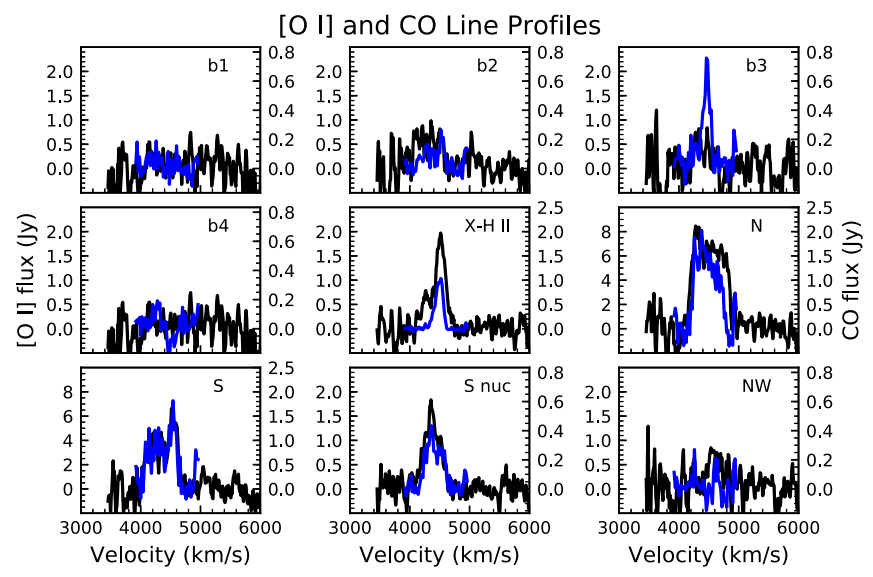

Figure 6. Line profiles for [O I] (black)and CO (1-0) (blue). The extraction regions are shown in Figure 1 and their positions and sizes are listed in Table 1.

range $0.1<R_{[\mathrm{C} \text { I] }}<0.5$, and is similar to that of NGC 6240 (Jiao et al. 2017). This latter system's value of $R_{[\mathrm{CI}]}=0.81 \pm$ 0.09 is unusual, even compared with most (U)LIRGs, and is believed to be primarily shock-heated. We can quantify this if the [C I] lines are optically thin. In that case the excitation temperature of the gas can be estimated from Walter et al. (2011) to be $T_{\mathrm{ex}}=38.8 \times\left[\ln \left(2.11 / R_{[\mathrm{C} \text { I }]}\right)\right]^{-1} \mathrm{~K}$, or $35 \mathrm{~K}$. This relatively high excitation temperature for [C I], and presumably CO (1-0) emitting molecular gas, is another indication of the unusual nature of the Taffy bridge, again suggesting shock heating as a viable mechanism for heating the gas.

Finally, extracting the $[\mathrm{C}$ II $] 157.7 \mu \mathrm{m}$ line flux from the same regions as the SPIRE SSW and SLW beams (5.4 and $22.0 \times 10^{-17} \mathrm{~W} \mathrm{~m}^{-2}$ respectively), yields $[\mathrm{C} \mathrm{II}] /[\mathrm{C} \mathrm{I}]=11$ (summing the fluxes from the $[\mathrm{C} \mathrm{I}](2-1)$ and $(1-0)$ transitions) and $[\mathrm{C} \mathrm{II}] /[\mathrm{N} \mathrm{II}]>54$. The value of $[\mathrm{CII}] /[\mathrm{CI}]$ is relatively low compared with values of $\sim 35$ for active star-forming galaxies (derived from results shown in Lu et al. 2015, 2017). This is consistent with the low measured $[\mathrm{C} \mathrm{II}] / \mathrm{CO}(1-0)$ ratios (see Section 3.4.1), since the [C I] and the CO (1-0) sample similar phases.

The nondetection of the $[\mathrm{N}$ II $] 205 \mu \mathrm{m}$ line, and a measured lower limit to the ratio of $[\mathrm{C} \mathrm{II}] /[\mathrm{N} \mathrm{II}]>54$ (where the $[\mathrm{C}$ II] is extracted from the larger SLW beam), implies a small 


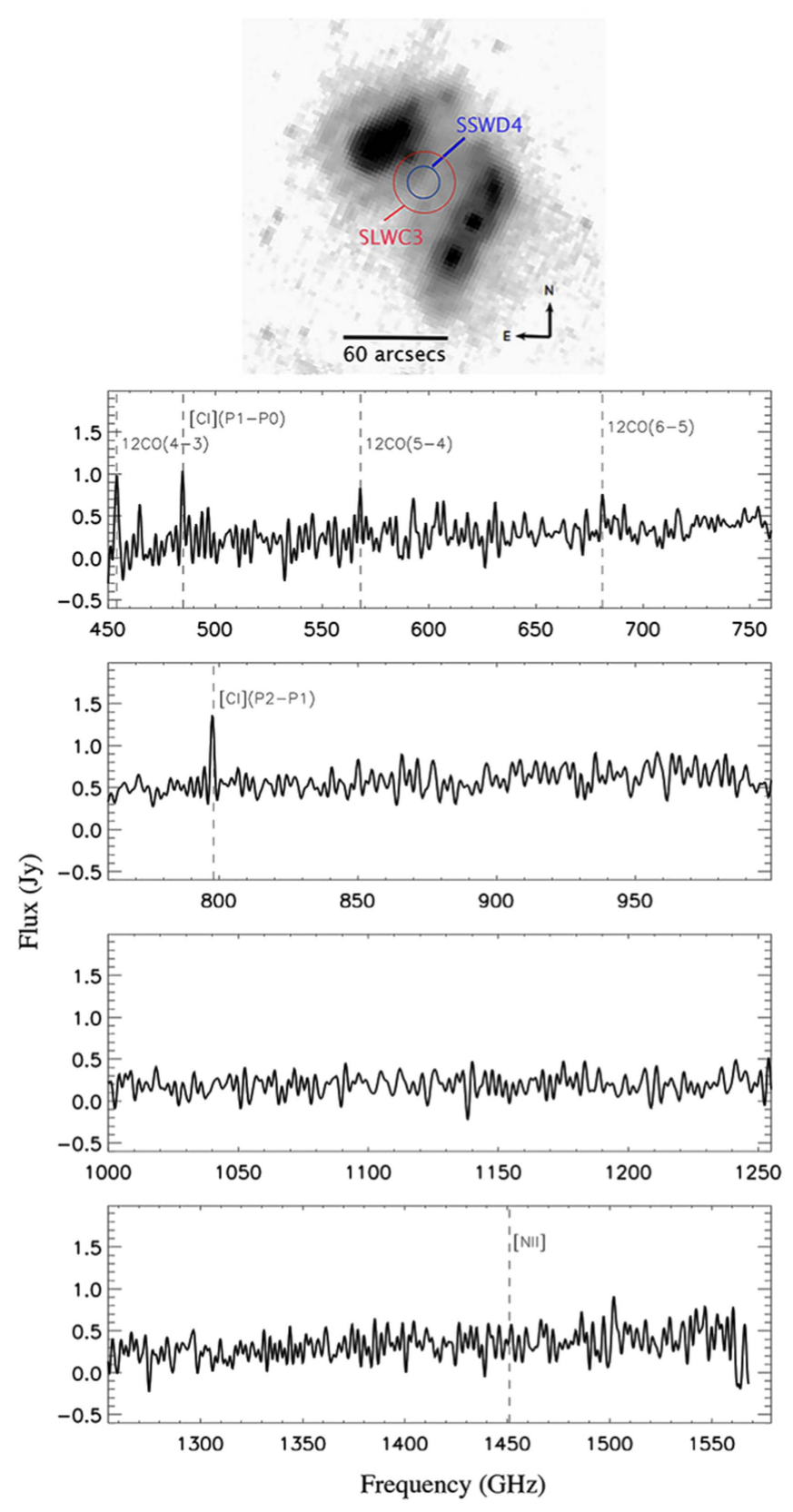

Figure 7. Top: $24 \mu \mathrm{m}$ image of Taffy with the beams for the SWS and SLW detector pointings shown. First three spectral panels: the SPIRE FTS spectrum from detector SLW C3, centered on the Taffy bridge, divided into three parts for clarity, showing the detection of ${ }^{12} \mathrm{CO}(6-5),{ }^{12} \mathrm{CO}(5-4)$, [ $\left.\mathrm{C} \mathrm{I}\right](1-0)$, and [C I] (2-1). The identification of the ${ }^{12} \mathrm{CO}(4-3)$ line is close to the band edge and should be treated with caution. The bottom panel shows part of the spectrum from the SSW-D4 detector (cospatial with SLW C3) spectrum that includes [N II] $205 \mu \mathrm{m}$, which is not detected. Extracted line fluxes and upper limits are given in Table 6.

ionized gas fraction in the bridge. For pure ionized gas, the ratio $[\mathrm{C} \mathrm{II}] /[\mathrm{N} \mathrm{II}] \sim 4$ and is almost independent of density. In normal galaxies, $[\mathrm{C} \mathrm{II}]$ emission arises primarily from the neutral gas phase, with typical values of $[\mathrm{C} \mathrm{II}] /[\mathrm{N}$ II] lying between 11 and 22 (Croxall et al. 2017, based on KINGFISH galaxy sample). The lower limit of this ratio for the Taffy bridge implies that most of the [C II] originates in a neutral or molecular phase.

\subsubsection{CO (1-0) versus [C II] Line Fluxes in the Taffy}

$\mathrm{CO}(1-0)$ line fluxes were extracted using the $\mathrm{CO}$ data cube of Gao et al. (2003); Table 5 shows the [C II]/CO ratios in the extraction regions. We find [C II]/CO ratios of $465 \pm 47$ and $670 \pm 67$ for UGC 12914 and UGC 12915, respectively. These values are much lower than the mean value for starburst galaxies, and lower than the values $\sim 6300$ found in galactic star formation regions by Stacey et al. (1991). They are also lower than the values typically found in normal galaxies $\sim 1500-3000$, with large scatter. The $[\mathrm{C} \mathrm{II}] / \mathrm{CO}$ ratios in the bridge are even lower, with $\mathrm{b} 2$ and $\mathrm{b} 3$ having ratios of $348 \pm 35$ and $218 \pm 22$, respectively. Low [C II]/CO values in normal spiral galaxies are often taken to imply that the galaxies are dominated by cool CO clouds with very little ambient UV radiation to excite [C II] emission over the same regions. The low value of $[\mathrm{C} \mathrm{II}] / \mathrm{CO}(1-0)$ is consistent with our earlier result that the $[\mathrm{CII}] /[\mathrm{CI}]$ ratio is also lower than that found in normal star-forming galaxies. This supports the earlier idea that the [C I] emission is probing the same gas phase as that of $\mathrm{CO}(1-0)$.

\section{The Origin of the $[\mathrm{C} \mathrm{II}]$ Emission in the Bridge}

\subsection{Star Formation in the Taffy System}

The SFRs for the two galaxy extraction regions estimated using MAGPHYS SED fits are $2.77 \pm 0.30$ and $1.13 \pm 0.01 M_{\odot} \mathrm{yr}^{-1}$ for UGC 12915 and 12914, respectively. These values are in good agreement with those of Appleton et al. (2015) who estimated the SFR using several different methods.

The SFR can also be estimated using the [C II] luminosities. Using the calibration of Herrera-Camus et al. (2015) with no IR color correction, we find SFRs of $5.2 M_{\odot} \mathrm{yr}^{-1}$ for UGC 12915 and $2.6 M_{\odot} \mathrm{yr}^{-1}$ for UGC 12914. For the two galaxies, the [C II]-based estimate is higher than the SED-based estimate by a factor of $\sim 2$. However, the IR color temperatures of both galaxies are on the lower end of the distribution for normal galaxies, and this would act to reduce the overall SFR derived from this correlation, bringing them into closer agreement with the SED method.

More interesting are the SFRs in the bridge and X-H II. Based on the SED fit, we estimate a total bridge SFR of $0.13 M_{\odot} \mathrm{yr}^{-1}$, excluding region $\mathrm{X}-\mathrm{H}$ II. This is in approximate agreement with estimates using the $24 \mu \mathrm{m}$ emission from the same regions (Appleton et al. 2015). On the other hand, if the [C II] line luminosity is used to calculate the SFR, we estimate a much higher value of $0.64 M_{\odot} \mathrm{yr}^{-1}$. Although there is a large spread in the SFR/[C II] calibration, this excess implies that the [C II] emission is boosted by processes other than normal star formation processes.

To explore this further, we attempt to quantify this excess in Figure 8 , where we plot the SFR surface density $\Sigma_{\mathrm{SFR}}$, determined from the Spitzer MIPS $24 \mu \mathrm{m}$ image of the system, with the $[\mathrm{C} \mathrm{II}]$ surface brightness $\Sigma_{[\mathrm{C} \mathrm{II}]}$. The figure is taken from Herrera-Camus et al. (2015), and includes a density distribution of points taken from a sample of 3486 regions in 46 normal galaxies. The two Taffy galaxies are marked as black circles in the diagram. The other extracted regions are marked in green. Except for the UGC 12914 nucleus, and region b4, most of the points, including the galaxies as a whole, lie on the extreme edge of the distribution for regions in normal galaxies. This makes the point that the $[\mathrm{C} \mathrm{II}]$ emission appears generally 
Table 6

SPIRE FTS Line Fluxes from Taffy Bridge ${ }^{a}$

\begin{tabular}{|c|c|c|c|c|c|}
\hline Line & $\begin{array}{c}\nu_{o b s} \\
(\mathrm{GHz})\end{array}$ & $\begin{array}{l}\text { FWHM }^{\mathrm{b}} \\
(\operatorname{arcsec})\end{array}$ & $\begin{array}{c}V_{\text {helio }}(\text { unc) } \\
\left(\mathrm{km} \mathrm{s}^{-1}\right)\end{array}$ & $\begin{array}{l}\text { Line Flux (unc) } \\
\left(\times 10^{-17} \mathrm{~W} \mathrm{~m}^{-2}\right)\end{array}$ & Detector \\
\hline $12 \mathrm{CO} 4-3$ & 453.93 & 43 & $4699 \pm 28$ & {$[1.33 \pm 0.09]^{\mathrm{c}}$} & SLWC3 \\
\hline $12 \mathrm{CO} 5-4$ & 567.80 & 35 & $4473 \pm 44$ & $0.68 \pm 0.08$ & SLWC3 \\
\hline $12 \mathrm{CO} 6-5$ & 681.09 & 32 & $4571 \pm 76$ & $0.33 \pm 0.09$ & SLWC3 \\
\hline [C I] $1-0$ & 484.74 & 38 & $4591 \pm 29$ & $1.20 \pm 0.09$ & SLWC3 \\
\hline [C I] 2-1 & 797.50 & 35 & $4452 \pm 29$ & $0.80 \pm 0.09$ & SLWC3 \\
\hline$[\mathrm{N} \mathrm{II}] 205 \mu \mathrm{m}$ & 1440.78 & 18 & $\ldots$ & $<0.08^{\mathrm{d}}$ & SSWD4 \\
\hline
\end{tabular}

Notes.

${ }^{\mathrm{a}}$ Observations centered on $\alpha(\mathrm{J} 2000)=00^{\mathrm{h}} 01^{\mathrm{m}} 40^{\mathrm{s}} \cdot 3, \delta(\mathrm{J} 2000)=23^{\mathrm{d}} 29^{\mathrm{m}} 22^{\mathrm{s}} \cdot 0$.

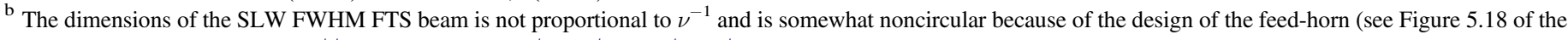
SPIRE Observer's Manual; http://herschel.esac.esa.int/Docs/SPIRE/html/spire_om.html).

${ }^{\mathrm{c}}$ Uncertain detection because the line is close to the SLW lower band edge.

d $3 \sigma$ upper limit.

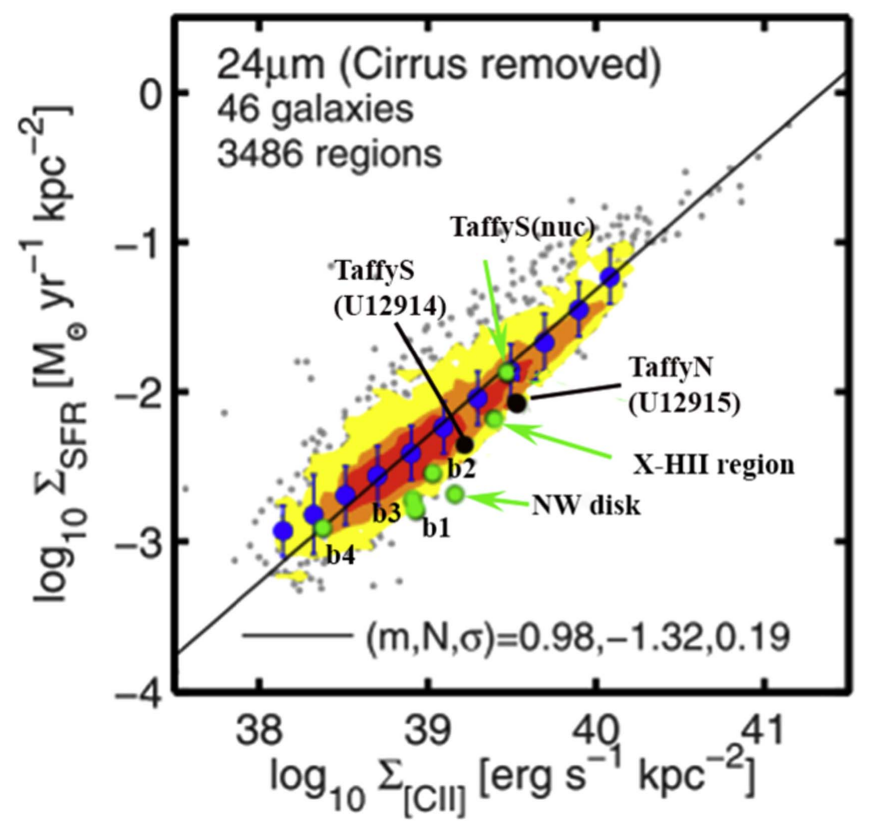

Figure 8. Star formation rate surface density, estimated from $24 \mu \mathrm{m}$ emission vs. [C II] surface brightness adapted from a figure by Herrera-Camus et al. (2015). The colored regions show the density of points from galaxy regions studied by Herrera-Camus et al. (2015). Black filled circles show the position on the same diagram of the Taffy galaxies, and the green filled circles show the bridge and other extracted regions from Figure 1. Note that many of the Taffy regions lie on the extreme edge of the distribution for normal galaxies, suggesting that the $[\mathrm{C} \mathrm{II}]$ emission may be boosted there.

boosted, compared with that expected for normal star formation. Alternatively, Figure 8 could be interpreted as showing lower SFR surface densities for a given [C II] surface density, compared with normal galaxies. However, as we shall see, it is more likely that the [C II] is boosted. We note that the region in the extreme northwest part of UGC 12915 (labeled NW disk in the figure) also shows unusually high [C II] emission. In this respect, it shows similarities with the bridge. Indeed, kinematically, it may be a projection of part of the northern bridge emission onto the galaxy (see Figures 2 and 3).

We can also compare the Taffy results with commonly measured properties of galaxies, such as the far-IR luminosity and color temperature. Figure 9 shows $L_{[\mathrm{C} \text { II }]} / L_{\mathrm{FIR}}$ plotted against $L_{\mathrm{FIR}}$ for the Taffy extraction regions. For comparison, we plot normal star-forming galaxies from Malhotra et al.

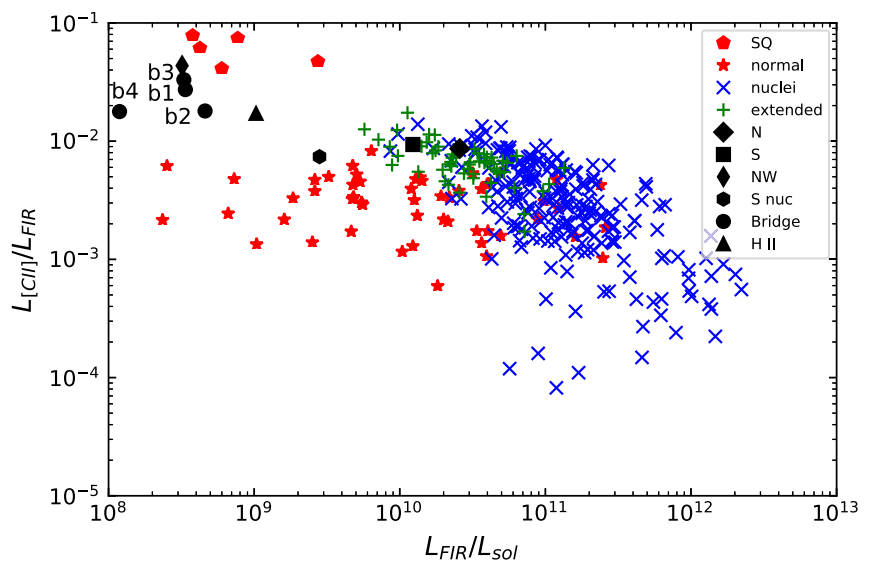

Figure 9. [C II]/FIR ratio for the bridge regions (black circles), extragalactic H II region (black triangle), southern galaxy (black square), southern nucleus (black hexagon), and northern galaxy (black diamond). For comparison, we show SQ (red pentagons; Appleton et al. 2013), normal galaxies from Malhotra et al. (2001; red stars), LIRG nuclei (Díaz-Santos et al. 2013; blue crosses), and LIRG extended emission (Díaz-Santos et al. 2014; green plus signs).

(2001) and regions from the group-wide shock in SQ (Appleton et al. 2013). We also show nuclei and extended emission from LIRGs in the GOALS sample (Díaz-Santos et al. 2013, 2014). The full-galaxy extractions and the UGC 12914 nucleus fall at the upper end of $L_{\text {[C II] }} / L_{\mathrm{FIR}}$ values for star-forming galaxies and LIRGs, so the emission from these regions is likely to be PDR-related. All of the bridge regions, the X-H II region, and the extended emission from the northwest region of UGC 12915 fall well above the distribution of normal galaxies, again supporting an enhancement in the [C II] bridge emission.

A similar conclusion is reached by plotting $L_{[\mathrm{C} \mathrm{II}]} / L_{\mathrm{FIR}}$ against the dust temperature as measured by $F_{60 \mu \mathrm{m}} / F_{100 \mu \mathrm{m}}$, as shown in Figure 10. Again, the full-galaxy extractions and UGC 12914 nucleus have $L_{[\mathrm{C} \mathrm{II}]} / L_{\mathrm{FIR}}$ ratios consistent with normal star-forming galaxies. The bridge regions, $\mathrm{X}-\mathrm{H}$ II, and northwest region of UGC 12915 are all well above the values of normal galaxies in the Malhotra et al. (2001) sample. The low $F_{60 \mu \mathrm{m}} / F_{100 \mu \mathrm{m}}$ temperatures in the bridge show that the dust is cool there, compared with warm gas temperatures. This is typical of shock-heated regions where the gas is heated more efficiently than the dust grains - a result that is contrary to that of a star-forming PDR, where much of the heating from young stars goes into warming the grains. 


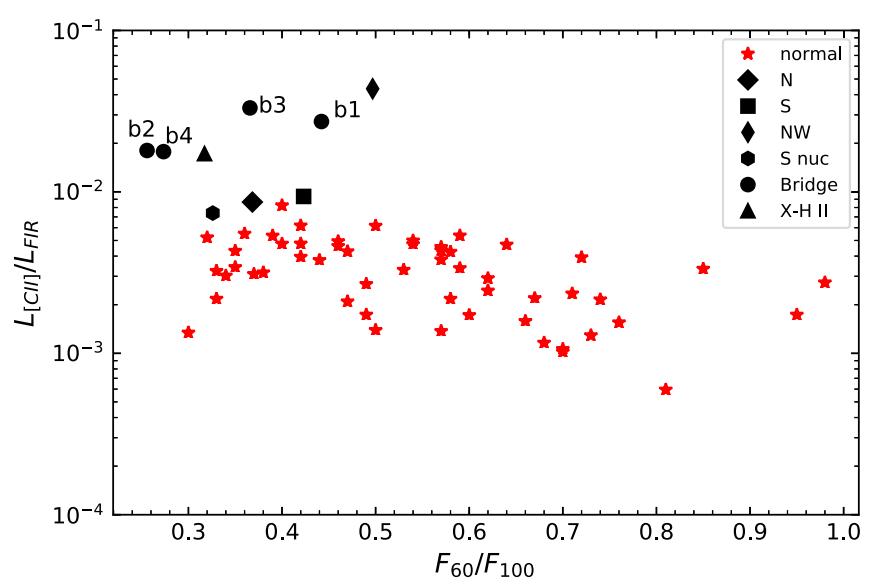

Figure 10. $[\mathrm{C} \mathrm{II}] / \mathrm{FIR}$ ratio vs. $F_{\nu}(60 \mu \mathrm{m}) / F_{\nu}(100 \mu \mathrm{m})$ (black circles), extragalactic $\mathrm{H}$ II region (black triangle), southern galaxy (black square), southern nucleus (black hexagon), and northern galaxy (black diamond). For comparison, we show normal galaxies from Malhotra et al. (2001; red stars).

\section{2. [C II] Boosting from Collisional Excitation with both $\mathrm{HI}$ and Warm $\mathrm{H}_{2}$}

Since the Taffy bridge contains large amounts of $\mathrm{HI}$, it is possible that $\mathrm{H}$ I could contribute to the collisional excitation of [C II] (Madden et al. 1997; Nikola et al. 1998). Indeed, as we have shown in Section 3.3, H I alone dominates the lowvelocity component of the bridge, with little detected $\mathrm{CO}$ emission at those velocities. Although we cannot rule out "dark" molecules at those velocities (Wolfire et al. 2010), it is worth exploring whether $\mathrm{H}$ I can provide part of the explanation for the [C II] emission. The surface intensity of [C II], $I_{C+}$ is given by the equation

$I_{C+}=h \nu A / 4 \pi\left[2 \mathrm{e}^{-91 / T} /\left(1+2 \mathrm{e}^{-91 / T}+n_{\mathrm{Hcr}} / n_{\mathrm{H}}\right)\right] \chi_{C+} \mathrm{N}_{\mathrm{H}} \Phi_{b}$,

where $h$ is Planck's constant, $A$ is the Einstein coefficient for the transition (we assume $2.29 \times 10^{-6} \mathrm{~s}^{-1}$ ), $T$ is the temperature, and $n_{\mathrm{H}}$ is the density of $\mathrm{HI}$. The critical density of $\mathrm{C}$ II for collisions with $\mathrm{HI}, n_{\mathrm{Hcr}}$, is a slowly decreasing function of temperature (Goldsmith et al. 2012), and is $\sim 3000 \mathrm{~cm}^{-3}$ at $T=100 \mathrm{~K} . \chi_{C+}$ is the fractional abundance of carbon. If the carbon is evenly split between the neutral and ionized forms, and assuming solar abundance, we adopt $\chi_{C+} \sim 1.5 \times 10^{-4} . N_{\mathrm{H}}$ is the column density of neutral hydrogen, and $\Phi_{b}$ is the beam filling factor, which we assume is unity. The assumption of solar abundance in the bridge is based on the belief that most of the gas in the bridge originated in the galaxies before the collision.

Clearly the density and temperature of the HI are not constrained by the observations, and so we use the equation to provide an order of magnitude estimate of the importance of collisions with $\mathrm{HI}$. If we take the peak value of $N_{\mathrm{H}}=1.13 \times 10^{21} \mathrm{~cm}^{-2}$ (Condon et al. 1993), $T=100 \mathrm{~K}$, $n_{\mathrm{H}}=100 \mathrm{~cm}^{-3}$ (assuming a cold neutral medium), and with the assumptions given above, we find $I_{C+}=0.98 \times$ $10^{-5} \mathrm{erg} \mathrm{s}^{-1} \mathrm{~cm}^{-2} \mathrm{sr}^{-1}$. This is close to the observed peak [C II] surface density in the bridge at position b3 of $1.3 \times$ $10^{-5} \mathrm{erg} \mathrm{s}^{-1} \mathrm{~cm}^{-2} \mathrm{sr}^{-1}$. Dropping the $\mathrm{H}$ I temperature to $50 \mathrm{~K}$ (given that the bridge is likely in adiabatic expansion as the galaxies pull away) would decrease $I_{C+}$ by a factor of 3 . Alternatively, increasing the $\mathrm{HI}$ density by a factor of two would more than double the value of $I_{C+}$. Given the large uncertainties in the many assumed quantities, including the filling factor, we can conclude that reasonable properties for the $\mathrm{HI}$ could potentially give rise to a significant fraction of the observed [C II] emission in the bridge.

However, we know that $\mathrm{HI}$ is not the only likely collisional partner with ionized carbon atoms. We already know from the previous Spitzer observations that the warm molecular gas is strongly radiating, so these molecules, which are likely to be shock-heated (Peterson et al. 2012), must also play a role in exciting the $[\mathrm{C} \mathrm{II}]$ transition. $^{25}$ Unlike the case of the $\mathrm{HI}$, we have better constraints on the properties of the warm $\mathrm{H}_{2}$ emission. For example, we know the temperature and column density of the bulk of the gas from fitting to the $\mathrm{H}_{2}$ excitation diagram for the pure rotational lines. Adopting a temperature for the warm $\mathrm{H}_{2}$ of $160 \mathrm{~K}$, a density of $1000 \mathrm{~cm}^{-3}$ (by analogy with the similar gas in SQ), a molecular column density of $3.2 \times 10^{20} \mathrm{~cm}^{-2}$, and a critical density of $5900 \mathrm{~cm}^{-3}$, the expected intensity of [C II] from this collisionally excited warm $\mathrm{H}_{2}$ would be $3.1 \times 10^{-5} \mathrm{erg} \mathrm{s}^{-1} \mathrm{~cm}^{-2} \mathrm{sr}^{-1}$. This is clearly more than enough to explain the observed [C II] intensity. We conclude that, within the considerable uncertainties, both $\mathrm{HI}$ and warm $\mathrm{H}_{2}$ are likely drivers of the enhanced [C II] emission seen in the bridge.

\section{Conclusions and Future Directions}

1. With the Herschel PACS and SPIRE spectrometers, we have mapped emission from [C II]157.7 $\mu \mathrm{m}$ and [O I] $63.2 \mu \mathrm{m}$ in both the galaxies and the bridge. In the bridge, we also detected [C I] $(2-1)$ and (1-0) emission, and $\mathrm{CO}$ emission lines from the high-J $\mathrm{CO}$ transition of $J=6-5,5-4$, and possibly $J=4-3$. The ratio of the [C I] lines suggests a gas temperature of $35 \mathrm{~K}$ for one component of the bridge, if these lines are optically thin. Taken together with the previous detection of a significant mass of warm $\mathrm{H}_{2}$ from pure rotational midIR emission from the bridge with higher temperatures $(150<\mathrm{T}<170 \mathrm{~K})$, and low dust temperatures, the results suggest that a warm, turbulent, multi-phase gaseous medium is present in the bridge that is the result of turbulent motions caused by the recent collision of the two galaxies.

2. The $[\mathrm{CII}]$ and $[\mathrm{OI}]$ emission in the bridge contains multiple kinematically broad components with intrinsic line widths of $150-250 \mathrm{~km} \mathrm{~s}^{-1}$ FWHM. The [C II] line shape in some parts of the bridge matches both the highand low-velocity components of the $\mathrm{HI}$, previously detected by the VLA, whereas the CO (1-0) line is most often associated with the highest-velocity [C II] component. This implies that the [C II]-emitting gas is distributed within both the $\mathrm{HI}$ and the $\mathrm{H}_{2}$ components in the bridge.

3. The $[\mathrm{C} \mathrm{II}]$ in the bridge (and part of the northern disk of UGC 12915) appears stronger than expected based on the very low levels of star formation observed there. For example, the [C II] emission in the bridge deviates from the relationships found between SFR, $L_{\mathrm{IR}}$ and [C II] emission in normal galaxies, suggesting an additional boosting of the emission.

\footnotetext{
There could also be an unknown quantity of colder "dark" molecules, which do not radiate strongly in $\mathrm{CO}$, but may be mixed with the $\mathrm{H}$. In the present paper, we do not include such a component.
} 
4. We explain the boosted values of [C II] emission in the bridge as due to a likely combination of both turbulently heated warm $\mathrm{H}_{2}$ (detected previously by Spitzer), as well as collisional excitation resulting from the large surface density of neutral hydrogen found in the bridge. With the current data, we cannot determine which of the two processes is dominant in the bridge, although both are likely to be important. Combined, these two collisional partners can excite the [C II]/FIR ratio to values as high as $3.3 \%$ in regions of the bridge. The failure to detect [N II] $205 \mu \mathrm{m}$ in the bridge to a low level, compared with the strength of the [C II] emission, rules out ionized gas (specifically electrons) as a significant factor in the excitation of the $[\mathrm{C} \mathrm{II}]$ transition.

The detection of [C II], [O I], and [C I] emission in the bridge presented here, taken together with the existence of powerful mid-IR warm $\mathrm{H}_{2}$ rotational emission from previous Spitzer observations, leads to the conclusion that the bridge contains several warm multi-phase gas components, despite having little ongoing star formation. In particular, the cooling time for the rotationally excited $\mathrm{H}_{2}$ is extremely short ( $\sim 1000$ years), and so shocks and turbulence must continue to inject energy into the gas to maintain the observed line luminosity (see Peterson et al. 2012). As we have shown, at least part of this energy helps to collisionally excite the [C II] emission.

One way in which the Taffy system differs from other known examples of turbulent energy dissipation in extragalactic systems, is that the main event that apparently triggered the formation of the bridge was the head-on collision between two gas-rich galaxies some $25 \mathrm{Myr}$ ago. In other examples of similar heating of gas via shocks and turbulence, the source of the turbulence continues to operate-for instance, the ongoing galaxy collision in $\mathrm{SQ}$, or the propagation of a radio jet into the ISM of a galaxy (Appleton et al. 2006; Ogle et al. 2007, 2010; Guillard et al. 2009; Cluver et al. 2010; Guillard et al. 2012b; Appleton et al. 2017). In the Taffy collision, how can this singular event continue to excite shocks in the bridge so long after the collision?

One solution might be large-scale coherent motions imparted on the gas at the time of the collision. Models of turbulent energy dissipation (e.g., Godard et al. 2014) suggest that a significant fraction of turbulent energy can be dissipated in large-scale vortices as well as compressive shocks. It is possible that the conditions in the head-on collision between two counter-rotating disks might have led to large-scale coherent vortices on the scale of a few kiloparsecs, which may be able to continue to drive shocks throughout the bridge. Although they do not discuss this specifically, the Vollmer et al. (2012) models show large-scale structure in the bridge that preserves some of the original rotational motion of the colliding galaxies. Preliminary results from our ALMA mapping of the $\mathrm{CO}$ emission in the Taffy bridge support this idea (P. N. Appleton et al. 2018, in preparation). If such largescale motions can last long enough, they could provide the source of the heating in the bridge, as density irregularities within large vortices could drive shocks into the surrounding lower-density material.

Further tests of this idea will require correlating regions of large-scale coherent motions with small-scale heating. The combination of millimeter telescope facilities like the NOEMA interferometer and ALMA, for mapping the detailed structure of the denser molecular gas on 100 pc to kiloparsec scales, with observations of warm molecular hydrogen with the James Webb Space Telescope, will likely provide new insight into how the gas is heated in the Taffy bridge and other similar systems. Furthermore, if small-scale shocks provide the main source of dissipation, at the end-point of a turbulent cascade, then the chemistry within the gas may show large-scale inhomogenities. For example, as shown by Godard et al. (2014), chemical reactions between $\mathrm{H}_{2}$ and [C II] ions can change the relative abundance of $[\mathrm{C} \mathrm{II}]$ and $\mathrm{CH}^{+}$. Systems like Taffy and SQ may be remarkable laboratories for future studies of how turbulence on kiloparsec scales can drive nonequilibrium thermal and chemical processes in diffuse intergalactic gas.

This work is based on observations made with Herschel, a European Space Agency Cornerstone Mission with significant participation by NASA. Support for this work was provided by NASA through an award issued by JPL/Caltech. B.W.P. also thanks the UW-BC Foundation for its generous support. U.L. acknowledges support by the research projects AYA2014-53506-P from the Spanish Ministerio de Economía y Competitividad, from the European Regional Development Funds (FEDER) and the Junta de Andalucía (Spain) grants FQM108. N.L. acknowledges support by National Key R\&D Program of China, \#2017YFA0402704 and NSFC \#11673028. Y.G. acknowledges support by National Key R\&D Program of China (2017YFA0402704), the NSFC \#11420101002 and the CAS Key Research Program of Frontier Sciences.

\section{ORCID iDs}

P. N. Appleton (1) https://orcid.org/0000-0002-7607-8766

T. Bitsakis (1) https://orcid.org/0000-0001-5787-8242

K. Alatalo ๑ https://orcid.org/0000-0002-4261-2326

M. Cluver (i) https://orcid.org/0000-0002-9871-6490

T. H. Jarrett (1) https://orcid.org/0000-0002-4939-734X

P. Ogle (1) https://orcid.org/0000-0002-3471-981X

\section{References}

Appleton, P. N., Guillard, P., Boulanger, F., et al. 2013, ApJ, 777, 66 Appleton, P. N., Guillard, P., Togi, A., et al. 2017, ApJ, 836, 76 Appleton, P. N., Lanz, L., Bitsakis, T., et al. 2015, ApJ, 812, 118 Appleton, P. N., Xu, K. C., Reach, W., et al. 2006, ApJL, 639, L51 Bakes, E. L. O., \& Tielens, A. G. G. M. 1994, ApJ, 427, 822 Braine, J., Davoust, E., Zhu, M., et al. 2003, A\&A, 408, L13 Bushouse, H. A. 1987, ApJ, 320, 49

Cluver, M. E., Appleton, P. N., Boulanger, F., et al. 2010, ApJ, 710, 248

Condon, J. J., Helou, G., Sanders, D. B., \& Soifer, B. T. 1993, AJ, 105, 1730 Cormier, D., Madden, S. C., Hony, S., et al. 2010, A\&A, 518, L57 Croxall, K. V., Smith, J. D., Pellegrinet, E., et al. 2017, ApJ, 845, 96 da Cunha, E., Charlot, S., \& Elbaz, D. 2008, MNRAS, 388, 1595 da Cunha, E., Charmandaris, V., Díaz-Santos, T., et al. 2010, A\&A, 523, 78 Dale, D. A., \& Helou, G. 2002, ApJ, 576, 159

Díaz-Santos, T., Armus, L., Charmandaris, V., et al. 2013, ApJ, 774, 68 Díaz-Santos, T., Armus, L., Charmandaris, V., et al. 2014, ApJL, 788, L17 Draine, B. T. 1978, ApJS, 36, 595

Fazio, G. G., Hora, J. L., Allen, L. E., et al. 2004, ApJS, 154, 10

Gao, Y., Zhu, M., \& Seaquist, E. R. 2003, AJ, 126, 2171

Glassgold, A. E., \& Langer, W. D. 1974, ApJ, 193, 73

Godard, B., Falgarone, E., \& Pineau des Forêts, G. 2014, A\&A, 570, A27 Goldsmith, P. F., Langer, W. D., Pineda, J. L., \& Velusamy, T. 2012, ApJS, 203, 13

Griffin, M. J., Abergel, A., Abreu, A., et al. 2010, A\&A, 518, L3

Guillard, P., Boulanger, F., Pineau des Forêts, G., et al. 2012a, ApJ, 749, 158 Guillard, P., Boulanger, F., Pineau Des Forêts, G., \& Appleton, P. N. 2009, A\&A, 502, 515

Guillard, P., Ogle, P. M., Emonts, B. H. C., et al. 2012b, ApJ, 747, 95 
Herrera-Camus, R., Bolatto, A. D., Wolfire, M. G., et al. 2015, ApJ, 800, 1 Hollenbach, D. J. 1989, in IAU Symp. 135, Interstellar Dust, ed. L. J. Allamandola \& A. G. G. M. Tielens (Cambridge: Cambridge Univ. Press), 227

Ingalls, J. G., Bania, T. M., Boulanger, F., et al. 2011, ApJ, 743, 174

Jarrett, T. H., Helou, G., Van Buren, D., Valjavec, E., \& Condon, J. J. 1999, AJ, 118, 2132

Jiao, Q., Zhao, Y., Zhu, M., et al. 2017, ApJL, 840, L18

Kennicutt, R. C., Jr. 1998, ApJ, 498, 541

Komugi, S., Tateuchi, K., Motohara, K., et al. 2012, ApJ, 757, 138

Lesaffre, P., Pineau Des Forets, G., Godard, B., et al. 2013, A\&A, 550, 106

Lu, N., Zhao, Y., Díaz-Santos, T., et al. 2017, ApJS, 230, 1

Lu, N., Zhao, Y., Xu, C. K., et al. 2015, ApJL, 802, L11

Lutz, D., Sturm, E., Genzel, R., et al. 2003, A\&A, 409, 867

Madden, S. C., Poglitsch, A., Geis, N., Stacey, G. J., \& Townes, C. H. 1997, ApJ, 483, 200

Malhotra, S., Kaufman, M. J., Hollenbach, D., et al. 2001, ApJ, 561, 766

Martin, D. C., Fanson, J., Schiminovich, D., et al. 2005, ApJL, 619, L1

Nikola, T., Genzel, R., Herrmann, F., et al. 1998, ApJ, 504, 749

O'Sullivan, E., Giacintucci, S., Vrtilek, J. M., Raychaudhury, S., \& David, L. P. 2009, ApJ, 701, 1560

Ogle, P., Antonucci, R., Appleton, P. N., \& Whysong, D. 2007, ApJ, 668, 699

Ogle, P., Boulanger, F., Guillard, P., et al. 2010, ApJ, 724, 1193

Papadopoulos, P. P., \& Greve, T. R. 2004, ApJL, 615, L29
Peterson, B. W., Appleton, P. N., Helou, G., et al. 2012, ApJ, 751, 11

Pilbratt, G. L., Riedinger, J. R., Passvogel, T., et al. 2010, A\&A, 518, L1

Poglitsch, A., Waelkens, C., Geis, N., et al. 2010, A\&A, 518, L2

Rieke, G. H., Young, E. T., Engelbracht, C. W., et al. 2004, ApJS, 154, 25

Roussel, H., Helou, G., Hollenbach, D. J., et al. 2007, ApJ, 669, 959

Soifer, B. T., Boehmer, L., Neugebauer, G., \& Sanders, D. B. 1989, AJ, 98,766

Stacey, G. J., Geis, N., Genzel, R., et al. 1991, ApJ, 373, 423

Struck, C. 1997, ApJS, 113, 269

Sturm, E., Bauer, O. H., Brauer, J., et al. 1998, in ASP Conf. Ser. 145, Astronomical Data Analysis Software and Systems VII, ed. R. Albrecht, R. N. Hook, \& H. A. Bushouse (San Francisco, CA: ASP), 161

Sulentic, J. W., Rosado, M., Dultzin-Hacyan, D., et al. 2001, AJ, 122, 2993

Tielens, A. G. G. M., \& Hollenbach, D. 1985, ApJ, 291, 747

Trinchieri, G., Sulentic, J., Pietsch, W., \& Breitschwerdt, D. 2005, A\&A, 444, 697

Vollmer, B., Braine, J., \& Soida, M. 2012, A\&A, 547, A39

Walter, F., Weiß, A., Downes, D., Decarli, R., \& Henkel, C. 2011, ApJ, 730,18

Watson, W. D. 1972, ApJ, 176, 271

Werner, M. W., Roellig, T. L., Low, F. J., et al. 2004, ApJS, 154, 1

Wolfire, M. G., Hollenbach, D., \& McKee, C. F. 2010, ApJ, 716, 1191

Xu, C. K., Lu, N., Condon, J. J., Dopita, M., \& Tuffs, R. J. 2003, ApJ, 595, 665

Zhu, M., Gao, Y., Seaquist, E. R., \& Dunne, L. 2007, AJ, 134, 118 